\title{
Adverse Gastrointestinal Effects with Kayexalate or Kalimate: A Comprehensive Review
}

This article was published in the following Dove Press journal:

Clinical and Experimental Gastroenterology

\author{
Yi-Hua Wu' \\ Jen-Wei Chou ${ }^{\text {I-4 }}$ \\ Hsiang-Chun Lai ${ }^{5}$ \\ Gin-Shen Su (D) \\ Ken-Sheng Cheng ${ }^{1,2}$ \\ Tsung-Wei Chen ${ }^{6}$ \\ 'Division of Gastroenterology and \\ Hepatology, Department of Internal \\ Medicine, China Medical University \\ Hospital, Taichung, Taiwan; ${ }^{2}$ School of \\ Medicine, China Medical University \\ Hospital, Taichung, Taiwan; ${ }^{3}$ Taiwan \\ Society of Inflammatory Bowel Disease, \\ Taipei, Taiwan; ${ }^{4}$ Taiwan Association for \\ the Study of Small Intestinal Diseases, \\ Taoyuan, Taiwan; ${ }^{5}$ Department of \\ Chinese Medicine, China Medical \\ University Hospital, Taichung, Taiwan; \\ ${ }^{6}$ Department of Pathology, Asia \\ University Hospital, Taichung, Taiwan
}

Correspondence: Jen-Wei Chou Division of Gastroenterology and Hepatology, Department of Interna Medicine. China Medical University Hospital, No. 2, Yude Road, North District, Taichung 40447, Taiwan

Tel $+886-4-22052121$ ext. 2220

Fax +886-4-22023II

Email codecol@yahoo.com.tw
Background: Patients with hyperkalemia are commonly treated with Kayexalate or Kalimate. Both drugs are associated with some fatal gastrointestinal (GI) adverse events (AEs).

Aim: To assess the clinical characteristics and outcomes of GI AEs induced by Kayexalate or Kalimate from published case reports.

Methods: We conducted a systematic review of case reports of Kayexalate or Kalimateinduced GI AEs, from PubMed, Medline, Cochrane Library, Clinical Key, and Google Scholar databases (1948 to March 31, 2020). We analyzed the clinical characteristics, GI AEs, and risk factors of enrolled patients.

Results: We identified 41 published articles describing 135 cases of GI AEs induced by Kayexalate (103 cases) or Kalimate (32 cases). The mean age of all patients was 55.5 years. Most patients were male $(54.8 \%)$. As high as $55.6 \%$ preparations were administered with sorbitol whereas $44.4 \%$ preparations had no sorbitol. The average time causing GI AEs was 19.8 days. Colon was the most commonly affected site (76.3\%). Drug crystals were histopathologically proven in $95.5 \%$ of the patients. Meanwhile, mortality was reported in $20.7 \%$.

Conclusion: Kayexalate or Kalimate, without or with sorbitol combination, may be related to fatal GI damage. Uremia, hypertension, and transplantation are predisposing factors. Clinicians should be careful in prescribing Kayexalate or Kalimate to patients.

Keywords: gastrointestinal injury, hyperkalemia, Kayexalate, Kalimate

\section{Introduction}

Patients suffering from chronic kidney disease (CKD) may exhibit hyperkalemia, a commonly seen adverse event of electrolyte imbalance that can lead to fatal cardiac arrhythmias. ${ }^{1}$ Hyperkalemia is commonly treated by a cation-exchange resin called sodium polystyrene sulfonate (Kayexalate). ${ }^{2-4}$ This drug acts as an exchange resin between sodium and potassium ions in the large intestine for accelerating potassium removal in the stool. ${ }^{5}$ However, its use has been reportedly related to colonic necrosis besides other fatal adverse events of gastrointestinal (GI) tract. ${ }^{6}$ An analog of Kayexalate, Kalimate (calcium polystyrene sulfonate), is also employed for treating hyperkalemia in clinical practice. However, there are not many incidences of Kalimate-induced GI tract injuries. ${ }^{7}$ Thus, our present study aimed to assess the clinical characteristics, risk factors, and outcomes of GI adverse events induced by Kayexalate or Kalimate from the published case reports in the literature. 


\section{Materials and Methods}

\section{Study Population}

We considered articles to be eligible that presented a case report or case series of GI adverse events associated with the use of Kayexalate or Kalimate. Any unfavorable or damaging effect on the GI tract in relation to the use of Kayexalate or Kalimate is considered as a GI adverse event. The age, sex, symptoms, GI injury location, and risk factors of enrolled patients were analyzed. If there is no information available for any case series from the original authors, those cases were excluded from this study.

\section{Literature Sources}

We searched databases including PubMed and Medline ( $\mathrm{n}$ =136), Cochrane Library $(\mathrm{n}=35)$, Clinical Key $(\mathrm{n}=203)$, and Google Scholar $(n=2134)$ (Figure 1. Flow diagram of included studies) (1948 to March 31, 2020) for the case reports, considered eligible, of GI adverse events associated with Kayexalate and Kalimate use. Languagerelated restrictions were not enforced in this study.

\section{Statistical Analysis}

The published reports contained 29 case reports, 11 case series, and 1 systematic review. The clinical characteristics of all enrolled patients were described using mean \pm standard deviation for continuous variables with abnormal distribution and number (percent) for categorical variables. Categorical variables were expressed as the number of cases and a percentage. These variables were compared using the $T$-test. A P value less than 0.05 indicates statistical significance.

\section{Ethics Considerations}

The present study was approved by the institutional review board of the Research Ethics Committee of China Medical University Hospital, in Taiwan (CMUH109-REC-01-049).

\section{Results}

A total of 2508 relevant articles were identified from our literature search. Following the application of the defined exclusion criteria, 41 articles describing 135 cases of GI adverse events induced by Kayexalate or Kalimate use

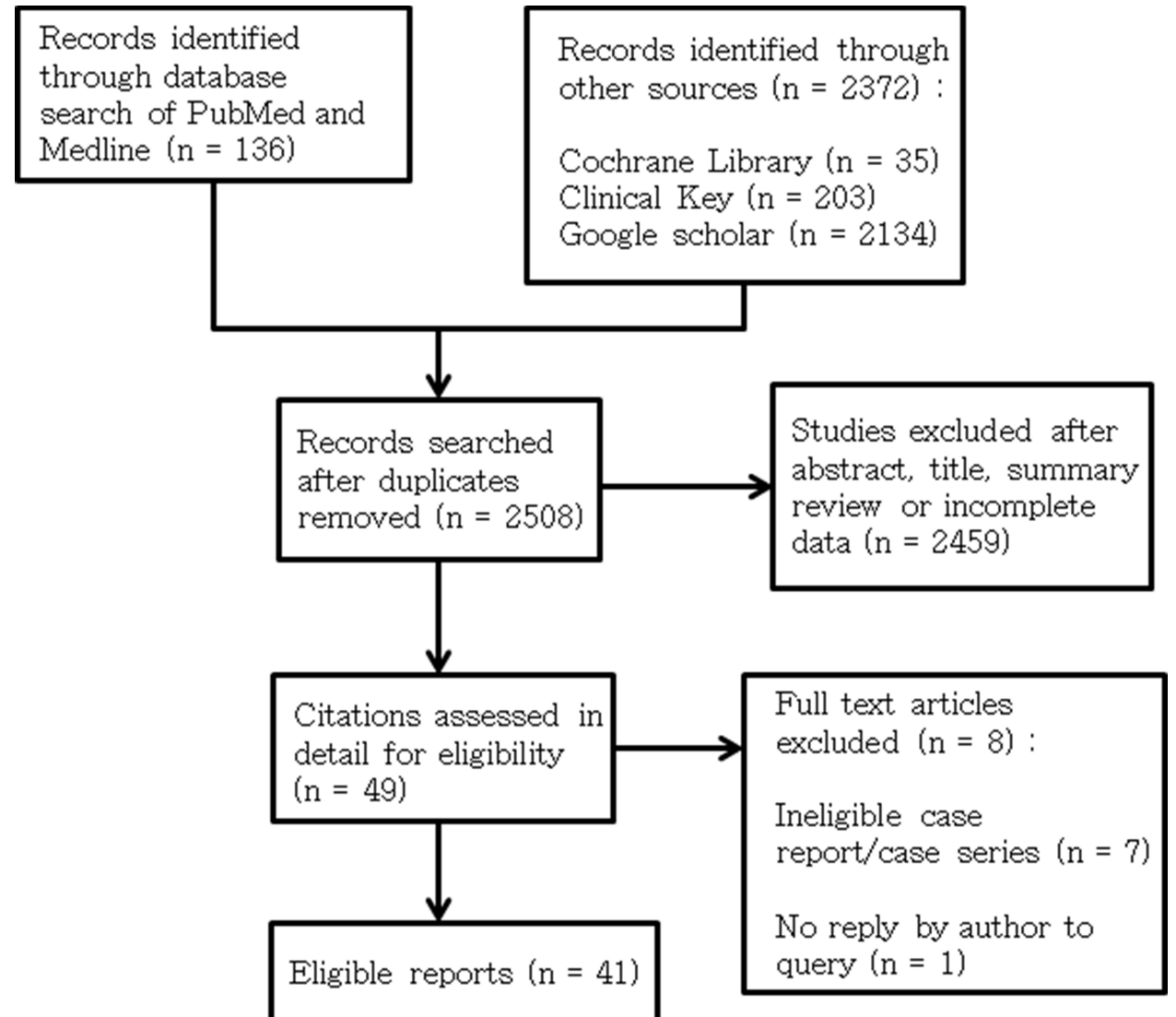

Figure I Flow diagram of included studies. 
were enrolled in our present study (Kayexalate: 103 cases; Kalimate: 32 cases). The enrolled patients were found in 29 case reports, 11 case series, and 1 systematic review (Figure 1). ${ }^{5-52}$ Three cases out of the 135 reported cases in our present study were reported before 1990, 24 cases were reported during 1990 to 2000, and another 108 cases were reported after 2000 .

Table 1 presents the clinical characteristics of all enrolled patients who experienced GI adverse events. The mean age of these patients was $55.5 \pm 20.2$ years (mean \pm standard deviation) with a range from 27 weeks of gestation to 91 years. In addition, $71.9 \%$ of them aged 55 years or more $(n=97)$, while $28.1 \%$ aged below 55 years $(\mathrm{n}=38)$. The differences between these two age groups were found to be statistically significant $(\mathrm{P}<$ 0.001 ). Regarding sex distribution, $54.8 \%$ of them were male $(n=74)$, whereas $45.2 \%$ were women $(n=61)$. The differences between the male and female groups were found to be statistically significant $(\mathrm{P}<0.001)$.

Moreover, $55.6 \%$ of all enrolled patients used Kayexalate or Kalimate with sorbitol concomitantly, whereas $44.4 \%$ used either of the drugs without sorbitol. A statistically significant difference was found between the sorbitol group and without-sorbitol group $(\mathrm{P}<0.001)$. In the analysis of predisposing factors for hyperkalemia, $87.7 \%$ had a history of CKD $(n=114)$ and $53.1 \%$ had endstage renal disease $(E S R D)$ requiring dialysis $(n=69)$, $30.0 \%$ had hypertension $(\mathrm{n}=39)$, and $18.5 \%$ had a prior solid transplant $(\mathrm{n}=24)$. The differences were statistically significant $(\mathrm{P}<0.001)$. The mean usage time of Kayexalate or Kalimate causing GI adverse events was 19.84 days ( 0.5 days to 1 year). Only 27 patients $(33.3 \%)$ received Kayexalate for more than 1 month. Kayexalate or Kalimate was administered orally in $67.8 \%$ of patients, rectally in $88.5 \%$, and via the nasogastric route in $2.5 \%$. The difference in administration routes was statistically significant $(\mathrm{P}<0.001)$.

The results of GI adverse events associated with Kayexalate or Kalimate use are summarized in Table 2 in our present study. The presenting GI symptoms of all enrolled patients were abdominal pain $(\mathrm{n}=59,68.6 \%)$, nausea or vomiting $(\mathrm{n}=6,7.0 \%)$, diarrhea $(\mathrm{n}=18$, $20.9 \%)$, and black stool or bloody stool $(\mathrm{n}=48,55.8 \%)$. A statistically significant difference was found between different symptoms $(\mathrm{P}<0.001)$. The colon was the most commonly involved site of the GI tract $(\mathrm{n}=103,76.3 \%)$, followed by the small intestine and duodenum (25.2\%), rectum (14.8\%), stomach $(7.4 \%)$ and esophagus $(3.0 \%)$.
The differences between these various sites were significant $(\mathrm{P}<0.001)$.

Histopathological findings associated with Kayexalate or Kalimate use were intestinal-wall ulcerations (83.7\%), necrosis $(60.0 \%)$, and perforation $(31.9 \%)$. The differences between these histological findings were statistically significant $(\mathrm{P}<0.001)$. In our present study, crystals of Kayexalate or Kalimate were demonstrated histopathologically in $95.5 \%$ of all patients. Meanwhile, mortality caused by GI injuries was reported in $20.7 \%$ of all patients in our present study.

\section{Discussion}

Kayexalate has been widely used for acute and chronic hyperkalemia management. It was subsequently approved by the FDA in the United States in $1958 .{ }^{7}$ Kalimate is a calcium-exchange resin with similar pharmacological action as that of Kayexalate, and it is also used for treating acute or chronic hyperkalemia. Kayexalate or Kalimate, which is a cation-exchange resin, can be introduced into the lower GI tract employing an enema preparation. This resin can also be introduced either orally or by nasogastric tube into the upper GI tract. When the resin is administered orally or by nasogastric tube into the stomach region, at first sodium or calcium cations are released from the resin in exchange with hydrogen ions in the stomach's acidic milieu. As the resin traverses the intestines, there is an exchange of hydrogen with potassium, followed by its excretion through the feces along with the remainder of the altered resin. Thus, the serum levels of potassium decrease over a period of hours to days. ${ }^{7,8}$

Conventionally, Kayexalate was given as a water suspension. Because of the concerns of fecal impaction and constipation, it became a common practice to administer Kayexalate together with hypertonic sorbitol. ${ }^{2}$ Sorbitol is a cathartic agent that has been reported to be associated with the development of intestinal injury rather than Kayexalate itself. The pathophysiological processes may be potentiated by the concomitant use of sorbitol with Kayexalate or Kalimate. Sorbitol, by elevating prostaglandin levels, causes a direct damage to the intestinal mucosa and leads to vasospasm and exacerbation of inflammation, and ultimately vascular injury. ${ }^{5}$ Histopathological lesions of these drugs-induced injuries vary from patchy inflammation to frank necrosis. ${ }^{8}$ This toxic effect of sorbitol on the GI tract has been proposed to potentially cause local ischemia and hypotension, in association with uremia and elevated renin levels in these patients, thereby adding to 


\begin{tabular}{|c|c|c|c|c|c|c|c|c|c|c|c|c|c|c|}
\hline & 气ू & 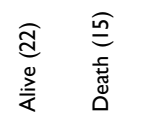 & 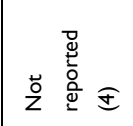 & & & & & 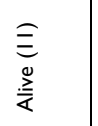 & 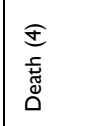 & 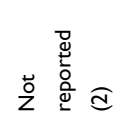 & & & & \\
\hline & 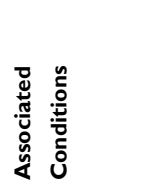 & 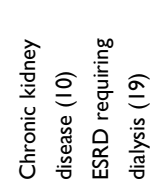 & 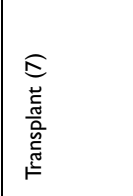 & 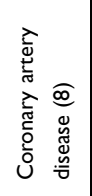 & $\begin{array}{l}0 \\
\vdots \\
0\end{array}$ & 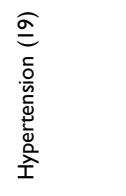 & 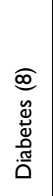 & 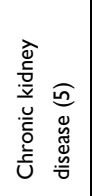 & 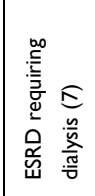 & 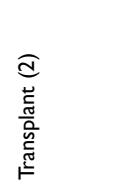 & 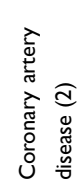 & $\stackrel{Ð}{Ð}$ & 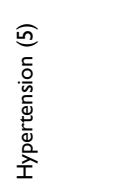 & 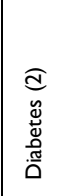 \\
\hline & 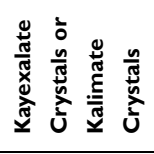 & $\stackrel{\infty}{m}$ & & & & & & \pm & & & & & & \\
\hline & 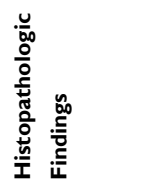 & 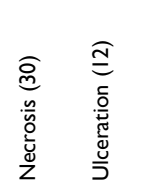 & 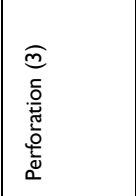 & & & & & 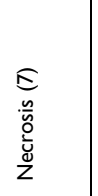 & 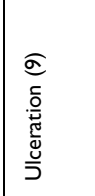 & $\begin{array}{l}\text { d } \\
\text { o } \\
\frac{0}{\pi} \\
\frac{0}{0} \\
\frac{0}{0} \\
0\end{array}$ & & & & \\
\hline & פ.ّ & 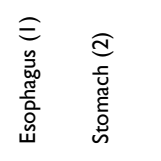 & 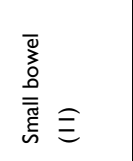 & 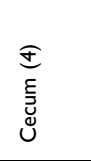 & 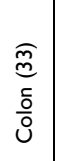 & 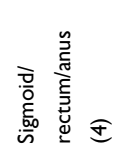 & & 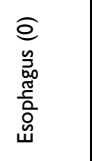 & 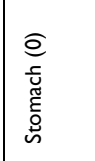 & 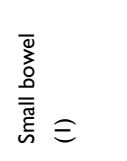 & $\begin{array}{l}\bar{a} \\
\underline{\bar{z}} \\
\text { Ũ }\end{array}$ & $\begin{array}{l}\widehat{\widehat{\Xi}} \\
\frac{\overline{0}}{0} \\
\mathcal{O}\end{array}$ & 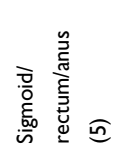 & \\
\hline & 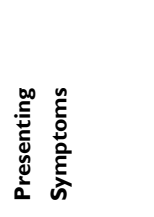 & 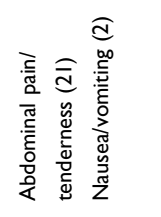 & $\begin{array}{l}\sigma \\
\bar{d} \\
\frac{d}{0} \\
\overline{0}\end{array}$ & 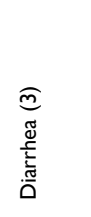 & & & & 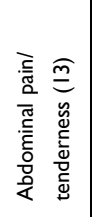 & 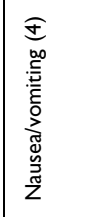 & $\begin{array}{l}\sqrt[n]{n} \\
\bar{d} \\
\frac{0}{0} \\
\bar{v}\end{array}$ & 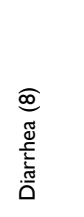 & & & \\
\hline & 宽 & $\underline{\underline{n}}$ & & & & & & in & & & & & & \\
\hline & 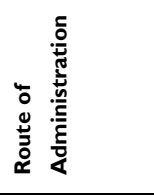 & 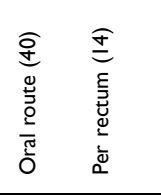 & 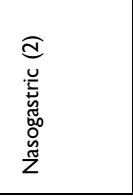 & & & & & 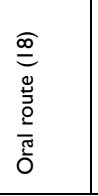 & 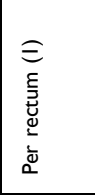 & 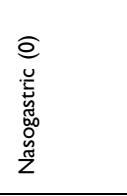 & & & & \\
\hline & 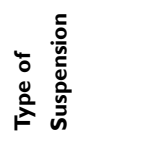 & 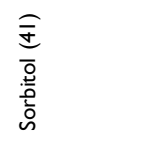 & & & & & & $\begin{array}{l}\overline{0} \\
\frac{0}{0} \\
\frac{0}{0} \\
\stackrel{0}{2} \subseteq\end{array}$ & & & & & & \\
\hline & 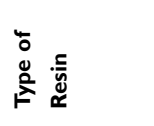 & 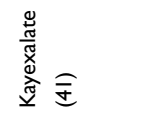 & & & & & & 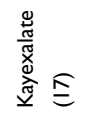 & & & & & & \\
\hline & 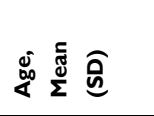 & $\begin{array}{l}\underline{\infty} \\
+1 \\
\infty \\
\infty\end{array}$ & & & & & & $\begin{array}{l}\stackrel{0}{+1} \\
\stackrel{+1}{n}\end{array}$ & & & & & & \\
\hline & ن⿺辶 & & $\frac{\frac{0}{\tilde{N}}}{\Sigma} \widehat{\mathbb{d}}$ & 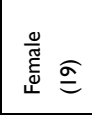 & & & & & & $\begin{array}{l}E \\
\frac{0}{N} \\
\frac{0}{\Sigma}\end{array}$ & 造 & & & \\
\hline & 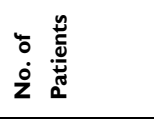 & $\stackrel{\infty}{\infty}$ & & & & & & & & & & & & \\
\hline & 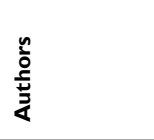 & 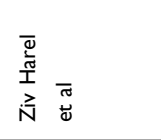 & & & & & & & & & & & & \\
\hline
\end{tabular}




\begin{tabular}{|c|c|c|c|c|c|c|c|c|c|c|c|c|c|c|c|c|c|c|}
\hline \multirow{2}{*}{ 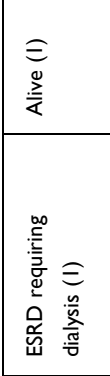 } & & \multirow{2}{*}{ 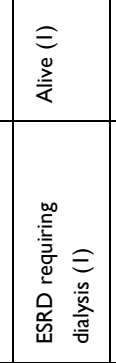 } & \multirow{2}{*}{\multicolumn{3}{|c|}{ 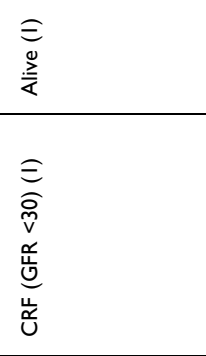 }} & \multicolumn{3}{|l|}{ 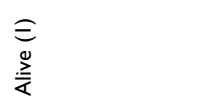 } & $\begin{array}{l}\widehat{\equiv} \\
\stackrel{\Xi}{\frac{\Xi}{\alpha}}\end{array}$ & \multicolumn{3}{|l|}{ 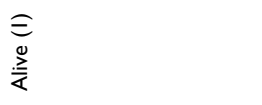 } & \multicolumn{2}{|l|}{ 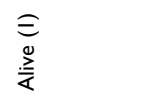 } & \multicolumn{4}{|l|}{ 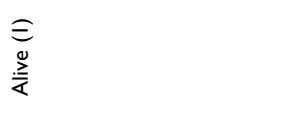 } \\
\hline & & & & & & 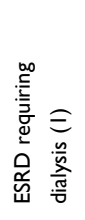 & 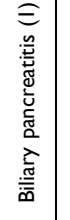 & 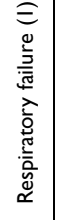 & 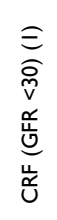 & & 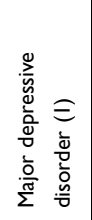 & 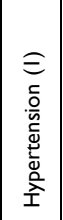 & 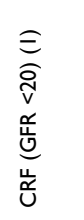 & 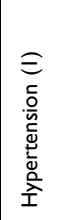 & 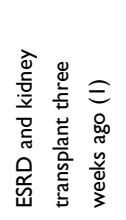 & 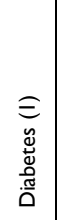 & 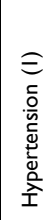 & 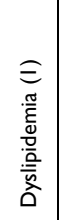 \\
\hline \multicolumn{2}{|l|}{-} & - & \multicolumn{3}{|l|}{-} & \multicolumn{3}{|l|}{-} & - & \multicolumn{3}{|l|}{-} & \multicolumn{3}{|l|}{-} & \multicolumn{3}{|l|}{-} \\
\hline $\begin{array}{l}\hat{\bar{a}} \\
\frac{n}{0} \\
\frac{0}{\dot{0}} \\
z \\
z\end{array}$ & 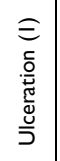 & 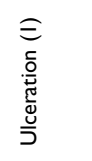 & 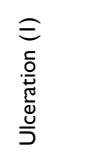 & & & 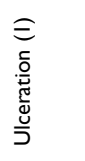 & 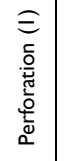 & & 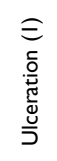 & 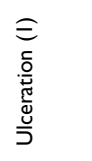 & & & 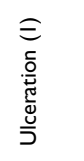 & 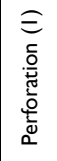 & 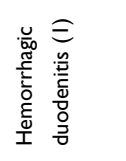 & 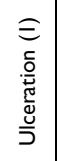 & & \\
\hline \multicolumn{2}{|l|}{$\begin{array}{l}\bar{\equiv} \\
\text { o. } \\
\text { O }\end{array}$} & 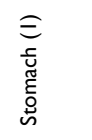 & 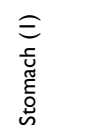 & 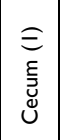 & 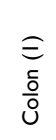 & \multicolumn{3}{|l|}{$\begin{array}{l}\bar{c} \\
\text { o. } \\
\text { O }\end{array}$} & 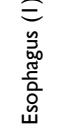 & \multicolumn{3}{|l|}{ 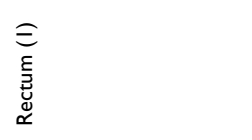 } & 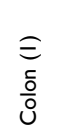 & & \multicolumn{4}{|l|}{ 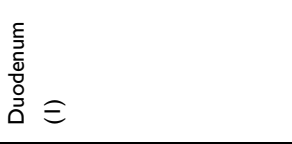 } \\
\hline 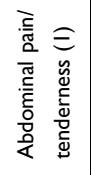 & $\begin{array}{l}\bar{\Xi} \\
\bar{d} \\
\frac{\mathbb{v}}{9} \\
\bar{v}\end{array}$ & $\begin{array}{l}\bar{\Xi} \\
\bar{d} \\
\frac{0}{0} \\
\overline{0}\end{array}$ & 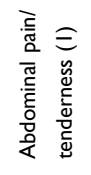 & 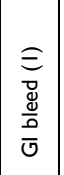 & & 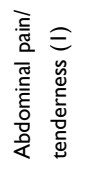 & 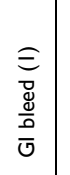 & & $\begin{array}{l}\widehat{\Xi} \\
\bar{d} \\
\frac{d}{0} \\
\bar{v}\end{array}$ & 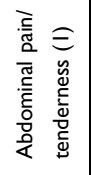 & $\begin{array}{l}\text { = } \\
\bar{d} \\
\frac{\mathbb{d}}{0} \\
\bar{u}\end{array}$ & 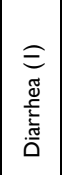 & 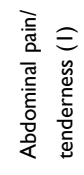 & & 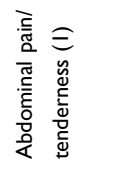 & 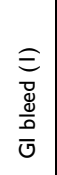 & & \\
\hline$\stackrel{\mathbb{z}}{\mathbf{z}}$ & & $\mathbb{z}$ & $\mathbb{z}$ & & & \pm & & & $\mathbb{z}$ & n & & & 8 & & \pm & & & \\
\hline 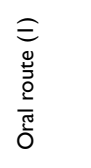 & & 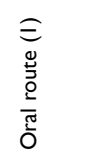 & 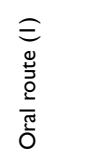 & & & 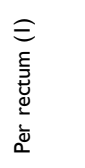 & & & 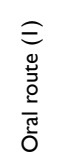 & 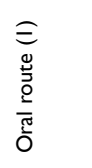 & & & 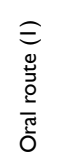 & & 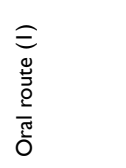 & & & \\
\hline $\begin{array}{l}\widehat{\equiv} \\
\text { o } \\
\frac{2}{0} \\
0 \\
0\end{array}$ & & $\begin{array}{l}\widehat{\Xi} \\
\overline{0} \\
\frac{0}{0} \\
\vdots \\
\vdots\end{array}$ & $\begin{array}{l}\widehat{\Xi} \\
\overline{0} \\
\frac{0}{0} \\
\vdots \\
\vdots\end{array}$ & & & $\begin{array}{l}\widehat{\equiv} \\
\overline{0} \\
\frac{0}{0} \\
\overline{0} \\
0\end{array}$ & & & $\begin{array}{l}\widehat{\equiv} \\
\overline{0} \\
\frac{0}{0} \\
0 \\
0\end{array}$ & 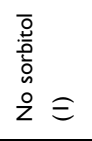 & & & $\begin{array}{l}\widehat{\equiv} \\
\overline{0} \\
\frac{0}{0} \\
\vdots \\
\vdots\end{array}$ & & $\begin{array}{l}\overline{0} \\
\frac{0}{0} \\
\frac{0}{0} \\
0 \\
2\end{array}$ & & & \\
\hline 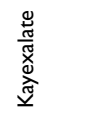 & & 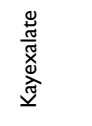 & 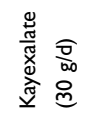 & & & 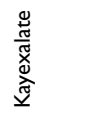 & & & 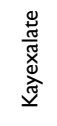 & 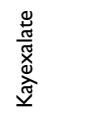 & & & 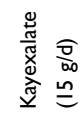 & & 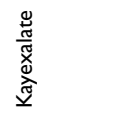 & & & \\
\hline 3 & & in & $\bar{\sigma}$ & & & $\stackrel{\infty}{n}$ & & & is & $\cong$ & & & $\overline{6}$ & & i̊ & & & \\
\hline 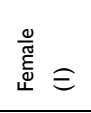 & & 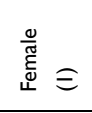 & $\begin{array}{l}\widehat{\Xi} \\
\frac{0}{\Sigma} \\
\frac{\pi}{\Sigma}\end{array}$ & & & $\begin{array}{l}\widehat{\Xi} \\
\frac{0}{\Sigma} \\
\frac{\pi}{\Sigma}\end{array}$ & & & $\begin{array}{l}\widehat{\equiv} \\
\frac{o}{\omega} \\
\frac{\omega}{\Sigma}\end{array}$ & 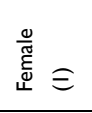 & & & $\begin{array}{l}\widehat{\Xi} \\
\frac{0}{\tilde{\pi}} \\
\Sigma\end{array}$ & & $\begin{array}{l}\widehat{\equiv} \\
\frac{0}{\tilde{\omega}} \\
\frac{\tilde{\omega}}{\Sigma}\end{array}$ & & & \\
\hline- & & - & - & & & - & & & - & - & & & - & & - & & & \\
\hline 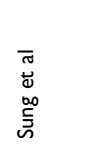 & & 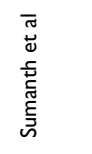 & 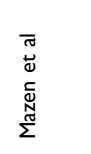 & & & 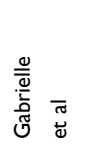 & & & $\begin{array}{l}\text { ब } \\
\stackrel{े}{0} \\
\stackrel{0}{E} \\
\frac{\bar{y}}{\bar{y}} \\
\Sigma\end{array}$ & 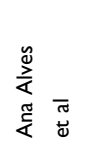 & & & 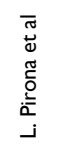 & & 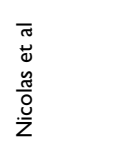 & & & \\
\hline
\end{tabular}




\begin{tabular}{|c|c|c|c|c|c|c|c|c|c|c|c|c|c|c|}
\hline 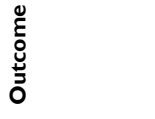 & $\begin{array}{l}\frac{a}{0} \\
\frac{0}{2} \\
\frac{2}{4}\end{array}$ & 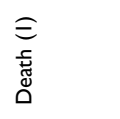 & \multicolumn{3}{|l|}{ 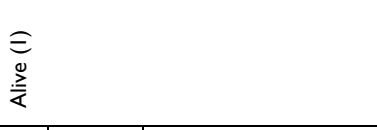 } & \multicolumn{4}{|l|}{$\begin{array}{l}\widehat{\Xi} \\
\text { 䓌 } \\
\text { D. }\end{array}$} & \multicolumn{2}{|l|}{ 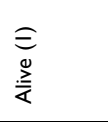 } & \multicolumn{3}{|l|}{$\begin{array}{l}\widehat{\Xi} \\
\frac{0}{\bar{\alpha}}\end{array}$} \\
\hline 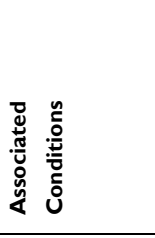 & 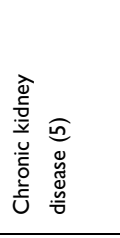 & 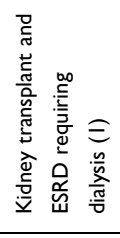 & 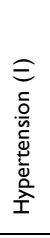 & 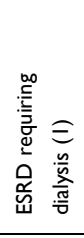 & 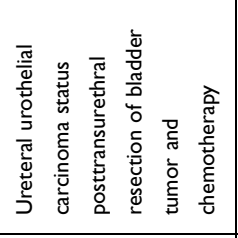 & 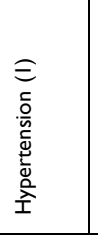 & 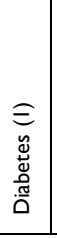 & 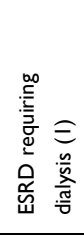 & 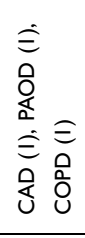 & 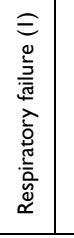 & 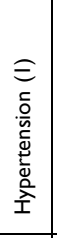 & 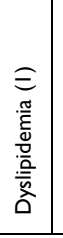 & 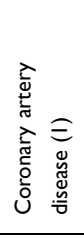 & 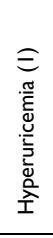 \\
\hline 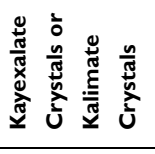 & \multicolumn{2}{|l|}{0} & \multicolumn{3}{|l|}{-} & \multicolumn{4}{|l|}{-} & \multicolumn{2}{|l|}{-} & \multicolumn{3}{|l|}{-} \\
\hline 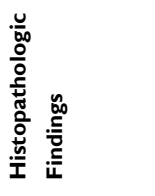 & 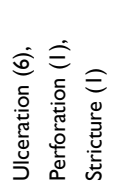 & 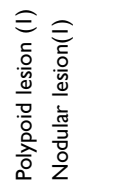 & \multicolumn{3}{|l|}{ 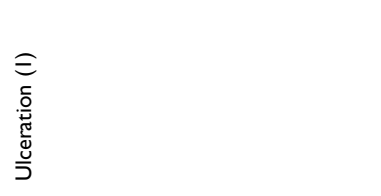 } & 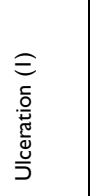 & 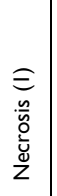 & 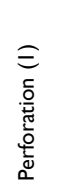 & & $\begin{array}{l}\widehat{0} \\
\frac{n}{\bar{u}} \\
\bar{u} \\
z \\
z\end{array}$ & 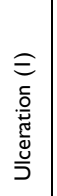 & \multicolumn{3}{|l|}{ 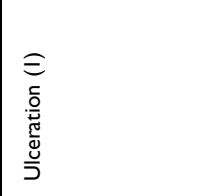 } \\
\hline פِّ & 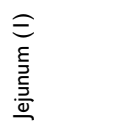 & 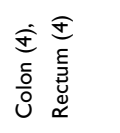 & \multicolumn{3}{|l|}{$\begin{array}{l}\hat{0} \\
\frac{0}{0} \\
\text { un }\end{array}$} & 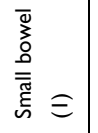 & $\begin{array}{l}\widehat{0} \\
\text { o } \\
\text { o }\end{array}$ & & & \multicolumn{2}{|c|}{ 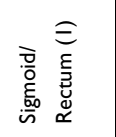 } & \multicolumn{3}{|l|}{ 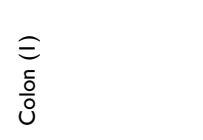 } \\
\hline 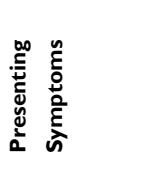 & 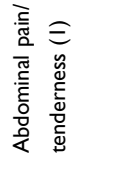 & $\begin{array}{l}\widehat{0} \\
\bar{d} \\
\frac{\mathbb{U}}{0} \\
\overline{0}\end{array}$ & \multicolumn{3}{|l|}{$\begin{array}{l}\equiv \\
\text { do } \\
\frac{0}{0} \\
\overline{0}\end{array}$} & 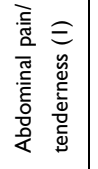 & 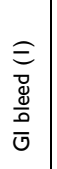 & & & $\begin{array}{l}\widehat{\Xi} \\
\bar{d} \\
\frac{d}{0} \\
\bar{v}\end{array}$ & & \multicolumn{3}{|l|}{$\begin{array}{l}\hat{\Xi} \\
\text { d } \\
\frac{\mathbb{d}}{0} \\
\bar{v}\end{array}$} \\
\hline 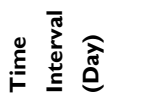 & \multicolumn{2}{|l|}{ n } & \multicolumn{3}{|l|}{$m$} & \multicolumn{4}{|l|}{$m$} & \multicolumn{2}{|l|}{$m$} & \multicolumn{3}{|l|}{$\mathbb{z}$} \\
\hline 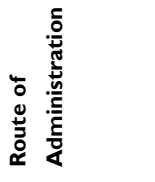 & \multicolumn{2}{|l|}{ 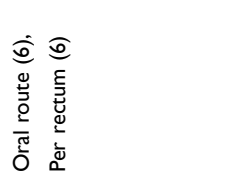 } & 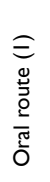 & 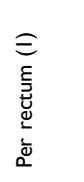 & & \multicolumn{4}{|l|}{ 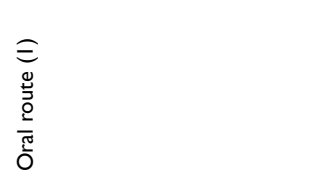 } & 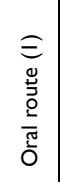 & 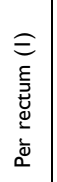 & \multicolumn{3}{|l|}{ 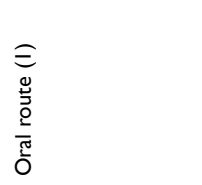 } \\
\hline 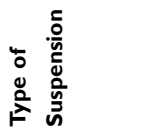 & \multicolumn{2}{|l|}{ 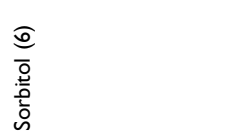 } & \multicolumn{3}{|l|}{ 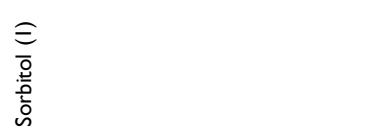 } & \multicolumn{4}{|l|}{$\begin{array}{l}\bar{o} \\
\frac{0}{0} \\
\overline{0} \\
o \\
\stackrel{2}{z}=\end{array}$} & \multicolumn{2}{|c|}{ 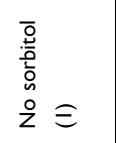 } & \multicolumn{3}{|c|}{$\begin{array}{l}\bar{g} \\
\frac{0}{0} \\
\overline{0} \\
\stackrel{0}{z}=\end{array}$} \\
\hline 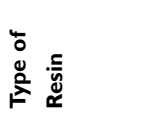 & \multicolumn{2}{|l|}{ 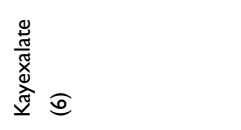 } & \multicolumn{3}{|l|}{ 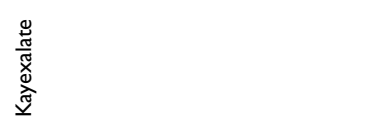 } & \multicolumn{4}{|l|}{ 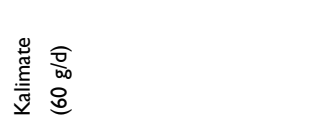 } & \multicolumn{2}{|l|}{ 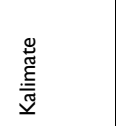 } & \multicolumn{3}{|l|}{ 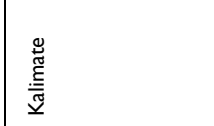 } \\
\hline 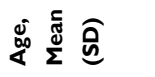 & \multicolumn{2}{|l|}{ อ } & $\stackrel{m}{\circ}$ & & & in & & & & $\dot{m}$ & & 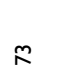 & & \\
\hline "ूँ & $\begin{array}{l}\frac{\Xi}{\Xi} \\
\frac{\sigma}{\Sigma} \\
\Sigma\end{array}$ & 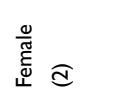 & $\begin{array}{l}\cong \\
\frac{0}{\tilde{\omega}} \\
\frac{\pi}{\Sigma}\end{array}$ & & & 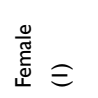 & & & & $\begin{array}{l}\cong \\
\frac{\omega}{\tilde{\omega}} \\
\frac{\omega}{\Sigma}\end{array}$ & & $\begin{array}{l}\cong \\
\frac{0}{\tilde{\omega}} \\
\frac{\sigma}{\Sigma}\end{array}$ & & \\
\hline 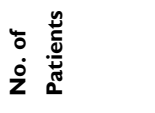 & $\circ$ & & - & & & - & & & & - & & - & & \\
\hline 号 & 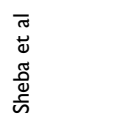 & & 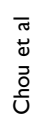 & & & 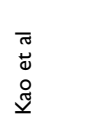 & & & & 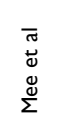 & & 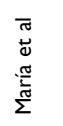 & & \\
\hline
\end{tabular}




\begin{tabular}{|c|c|c|c|c|c|c|c|c|c|c|c|c|c|c|c|c|c|}
\hline \multicolumn{3}{|l|}{$\begin{array}{l}\widehat{=} \\
\stackrel{0}{\frac{\pi}{4}}\end{array}$} & \multicolumn{3}{|l|}{ 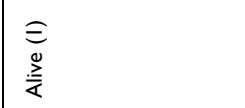 } & \multicolumn{3}{|l|}{ 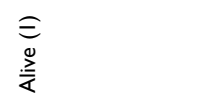 } & \multirow{2}{*}{ 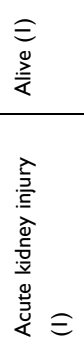 } & \multirow{2}{*}{\multicolumn{2}{|c|}{ 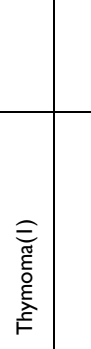 }} & \multicolumn{3}{|l|}{$\begin{array}{l}\widehat{\Xi} \\
\text { 吉 } \\
\text { Dू }\end{array}$} & \multicolumn{3}{|l|}{ 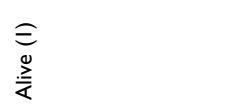 } \\
\hline 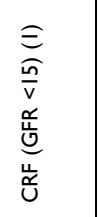 & 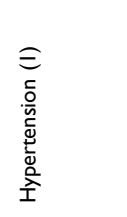 & & 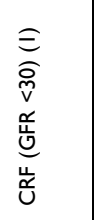 & 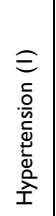 & & 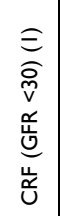 & 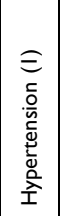 & 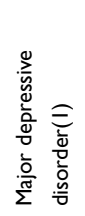 & & & & 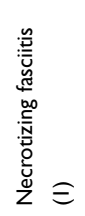 & 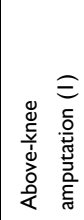 & 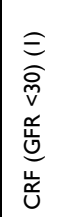 & 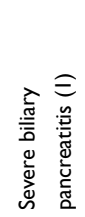 & 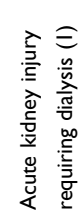 & 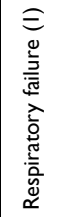 \\
\hline \multicolumn{3}{|l|}{ - } & \multicolumn{3}{|l|}{-} & \multicolumn{3}{|l|}{-} & \multicolumn{3}{|l|}{-} & \multicolumn{3}{|l|}{$\circ$} & \multicolumn{3}{|l|}{ - } \\
\hline 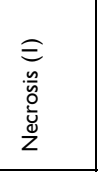 & 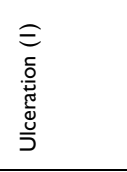 & 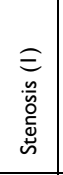 & 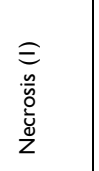 & 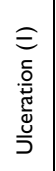 & & 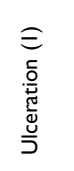 & & & 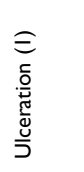 & & & 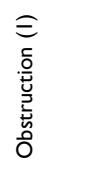 & & & 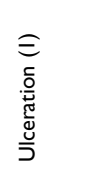 & 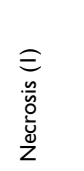 & 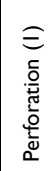 \\
\hline $\begin{array}{l}\widehat{\Xi} \\
\frac{0}{0} \\
\text { o }\end{array}$ & 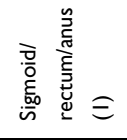 & & 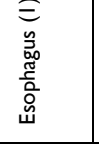 & 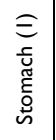 & 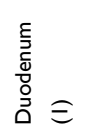 & 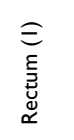 & & & 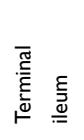 & 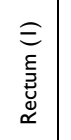 & $\mid \begin{array}{l}0 \\
\bar{c} \\
0 \\
0 \\
\end{array}$ & 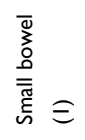 & 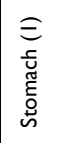 & $\begin{array}{l}\hat{E} \\
\text { o. } \\
0\end{array}$ & $\begin{array}{l}\widehat{0} \\
\frac{\delta}{0} \\
\text { U }\end{array}$ & & \\
\hline 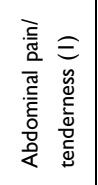 & 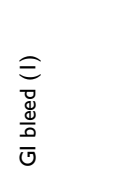 & & 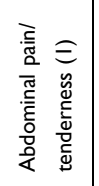 & $\begin{array}{l}\widehat{\Xi} \\
\bar{\Xi} \\
\frac{\mathscr{U}}{0} \\
\bar{U}\end{array}$ & & 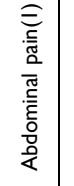 & 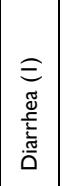 & & 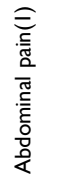 & 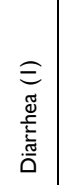 & $\begin{array}{l}\widehat{\Xi} \\
\bar{d} \\
\frac{\delta}{0} \\
\overline{0}\end{array}$ & 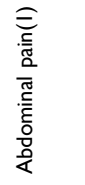 & 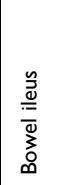 & & $\begin{array}{l}\bar{\Xi} \\
\bar{\Xi} \\
\frac{\mathrm{d}}{0} \\
\bar{U}\end{array}$ & & \\
\hline$\stackrel{\beth}{ }$ & & & \pm & & & N & & & ナ & & & in & & & \pm & & \\
\hline 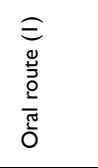 & & & 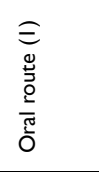 & & & 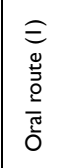 & & & 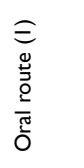 & & & 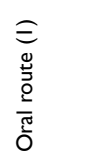 & & & 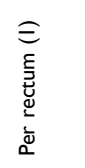 & & \\
\hline $\begin{array}{l}\overline{0} \\
\frac{9}{0} \\
\frac{0}{0} \\
0 \\
0 \\
z\end{array}$ & & & $\begin{array}{l}\overline{8} \\
\frac{0}{0} \\
\overline{0} \\
\stackrel{0}{z} \cong\end{array}$ & & & 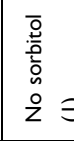 & & & 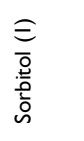 & & & 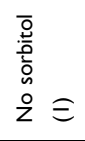 & & & 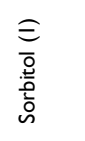 & & \\
\hline 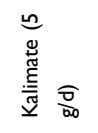 & & & 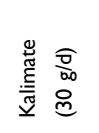 & & & 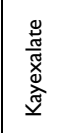 & & & 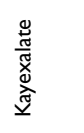 & & & $\begin{array}{l}\stackrel{\Xi}{\tilde{\Xi}} \\
\underline{\underline{\underline{E}}} \\
\underline{\underline{E}}\end{array}$ & & & 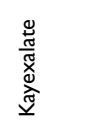 & & \\
\hline$\stackrel{\circ}{\circ}$ & & & R & & & œ & & & ஜ & & & $\tilde{n}$ & & & $\stackrel{\infty}{\infty}$ & & \\
\hline 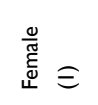 & & & 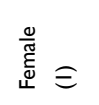 & & & $\stackrel{\frac{0}{\widetilde{E}}}{\underset{\Psi}{\tilde{E}}}$ & & & 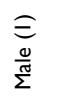 & & & 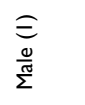 & & & 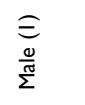 & & \\
\hline- & & & - & & & - & & & - & & & - & & & - & & \\
\hline $\begin{array}{l} \\
\bar{\sigma} \\
\stackrel{5}{0} \\
\bar{\Xi}\end{array}$ & & & $\begin{array}{l}\bar{\Xi} \\
\bar{\Xi} \\
\bar{\xi}\end{array}$ & & & 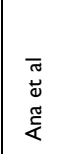 & & & 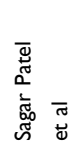 & & & 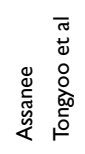 & & & 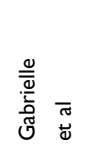 & & \\
\hline
\end{tabular}




\begin{tabular}{|c|c|c|c|c|c|c|c|c|c|c|c|c|c|c|c|}
\hline 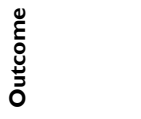 & \multicolumn{3}{|l|}{$\begin{array}{l}\hat{\overline{0}} \\
\hat{\frac{0}{\alpha}}\end{array}$} & \multicolumn{2}{|l|}{$\begin{array}{l}\text { d } \\
\frac{0}{2} \\
\frac{2}{4}\end{array}$} & \multicolumn{2}{|l|}{ 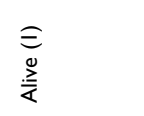 } & \multicolumn{3}{|l|}{$\begin{array}{l}\widehat{\Xi} \\
\stackrel{0}{\frac{2}{\alpha}}\end{array}$} & \multicolumn{5}{|l|}{$\begin{array}{l}\widehat{\Xi} \\
\stackrel{0}{\frac{\pi}{<}}\end{array}$} \\
\hline 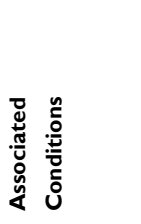 & 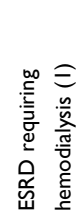 & 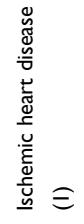 & 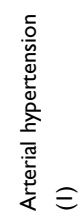 & 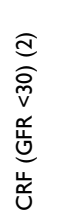 & & 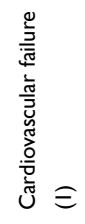 & 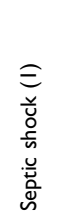 & 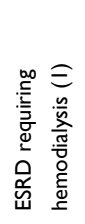 & 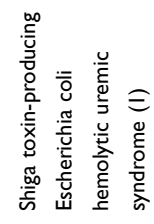 & & $\begin{array}{l}\hat{\equiv} \\
z \\
\text { 至 }\end{array}$ & $\begin{array}{l}\widehat{E} \\
\Sigma \\
\Delta\end{array}$ & $\begin{array}{l}\widehat{\Xi} \\
\text { 岌 } \\
\text { U }\end{array}$ & $\widehat{\Xi}$ & $\begin{array}{l}\text { 三 } \\
\text { ò }\end{array}$ \\
\hline 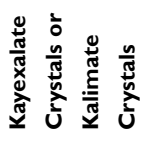 & \multicolumn{3}{|l|}{-} & \multicolumn{2}{|l|}{$\sim$} & \multicolumn{2}{|l|}{-} & \multicolumn{3}{|l|}{-} & \multicolumn{5}{|l|}{-} \\
\hline 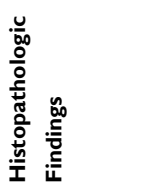 & 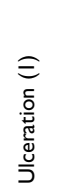 & 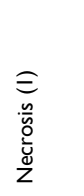 & & 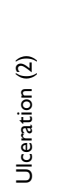 & & 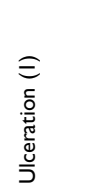 & $\begin{array}{l}\widehat{0} \\
\frac{n}{\bar{u}} \\
\overline{\underline{u}} \\
z\end{array}$ & $\begin{array}{l}\widehat{\Xi} \\
\overline{\grave{\Xi}} \\
\bar{J}\end{array}$ & 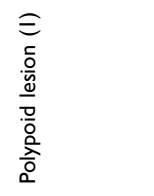 & & 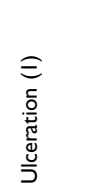 & 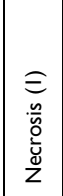 & 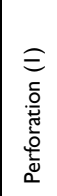 & & \\
\hline وَّ & \multicolumn{3}{|l|}{$\begin{array}{l}0 \\
\bar{o} \\
\text { o } \\
0\end{array}$} & $\begin{array}{l}a \\
\frac{0}{0} \\
\frac{0}{0}\end{array}$ & 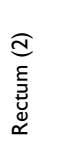 & \multicolumn{2}{|l|}{ 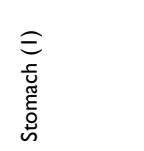 } & 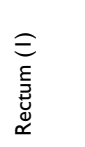 & 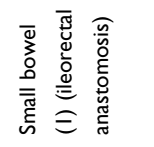 & $\begin{array}{l}\hat{E} \\
\overline{0} \\
0\end{array}$ & \multicolumn{5}{|l|}{$\begin{array}{l}0 \\
\bar{o} \\
\frac{\delta}{0}\end{array}$} \\
\hline 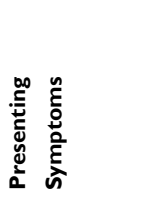 & \multicolumn{3}{|l|}{ 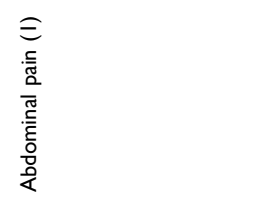 } & \multicolumn{2}{|l|}{$\begin{array}{l}\widehat{\equiv} \\
\bar{d} \\
\frac{\mathrm{d}}{\mathrm{a}} \\
\overline{\mathrm{U}}\end{array}$} & $\begin{array}{l}0 \\
\bar{d} \\
\frac{\Phi}{0} \\
\overline{0}\end{array}$ & & \multicolumn{3}{|l|}{$\begin{array}{l}\widehat{\Xi} \\
\bar{d} \\
\frac{\Phi}{0} \\
\bar{v}\end{array}$} & 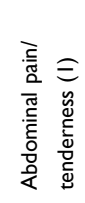 & $\begin{array}{l}\widehat{\Xi} \\
\bar{d} \\
\frac{\mathbb{d}}{0} \\
\overline{0}\end{array}$ & & & \\
\hline 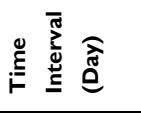 & \multicolumn{3}{|l|}{$m$} & \multicolumn{2}{|l|}{ N } & \multicolumn{2}{|l|}{ N } & \multicolumn{3}{|l|}{ - } & \multicolumn{5}{|l|}{ n } \\
\hline 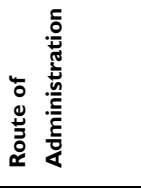 & \multicolumn{3}{|l|}{ 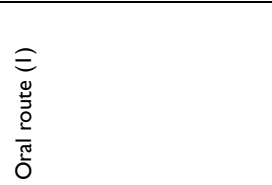 } & \multicolumn{2}{|l|}{ 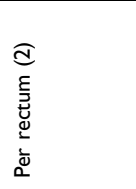 } & 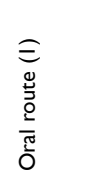 & & \multicolumn{3}{|l|}{ 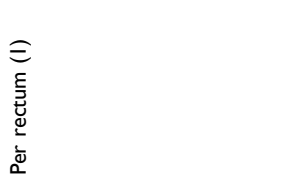 } & \multicolumn{5}{|l|}{ 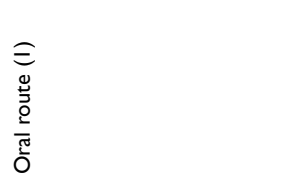 } \\
\hline 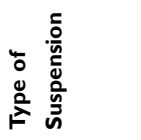 & \multicolumn{3}{|l|}{ 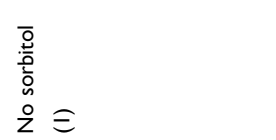 } & \multicolumn{2}{|c|}{ 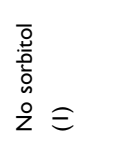 } & $\begin{array}{l}\widehat{\bar{a}} \\
\overline{\frac{9}{20}} \\
\frac{0}{0}\end{array}$ & & \multicolumn{3}{|l|}{$\begin{array}{l}\widehat{\bar{o}} \\
\frac{0}{20} \\
\frac{0}{0} \\
0\end{array}$} & \multicolumn{4}{|l|}{ 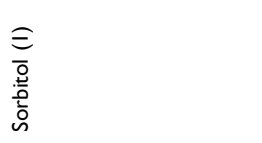 } & \\
\hline 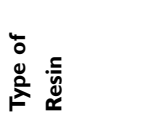 & \multicolumn{3}{|l|}{ 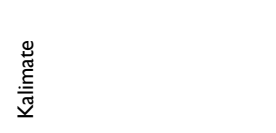 } & & 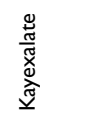 & & \multicolumn{2}{|l|}{ 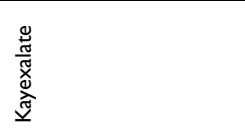 } & & \multicolumn{5}{|l|}{ 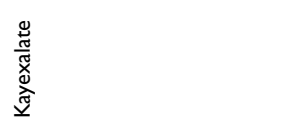 } \\
\hline 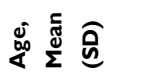 & \multicolumn{3}{|l|}{$\approx$} & $\stackrel{+}{m}$ & t & 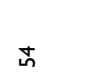 & & $\sigma$ & & & : & & & & \\
\hline 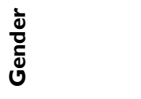 & $\begin{array}{l}\widehat{\equiv} \\
\frac{0}{\tilde{\omega}} \\
\frac{\pi}{\Sigma}\end{array}$ & & & 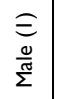 & 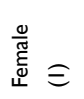 & 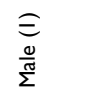 & & 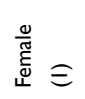 & & & 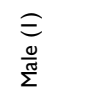 & & & & \\
\hline 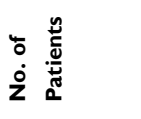 & - & & & $\sim$ & & - & & - & & & - & & & & \\
\hline 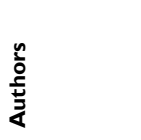 & 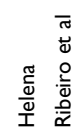 & & & 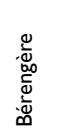 & & 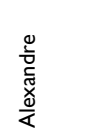 & & 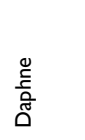 & & & 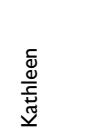 & & & & \\
\hline
\end{tabular}




\begin{tabular}{|c|c|c|c|c|c|c|c|c|c|c|c|c|c|c|c|}
\hline \multicolumn{3}{|l|}{$\begin{array}{l}\text { હ } \\
\text { 䓌 } \\
\text { ô } \\
\end{array}$} & \multicolumn{3}{|l|}{$\begin{array}{l}\bar{\equiv} \\
\text { 离 } \\
\text { o. }\end{array}$} & \multicolumn{2}{|l|}{$\begin{array}{l}\widehat{\equiv} \\
\substack{\frac{0}{<} \\
\frac{\pi}{<}}\end{array}$} & \multicolumn{2}{|l|}{ 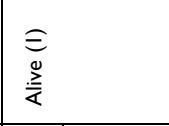 } & \multicolumn{3}{|l|}{$\begin{array}{l}\widehat{\equiv} \\
\stackrel{0}{\frac{2}{\alpha}}\end{array}$} & \multicolumn{3}{|l|}{ 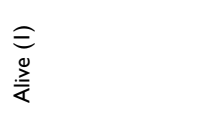 } \\
\hline $\begin{array}{l}\overline{\overline{0}} \\
\text { 号 } \\
\text { un }\end{array}$ & $\begin{array}{l}\text { 三 } \\
\text { ż } \\
\text { I }\end{array}$ & $\begin{array}{l}\hat{\Xi} \\
\Sigma \\
\overline{0}\end{array}$ & 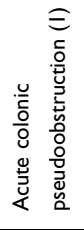 & $\begin{array}{l}\widehat{\equiv} \\
\frac{\bar{v}}{\alpha}\end{array}$ & & 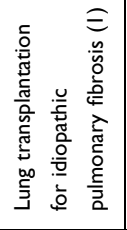 & 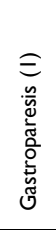 & 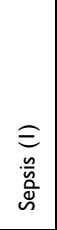 & $\begin{array}{l}\hat{\overline{0}} \\
0 \\
\text { 岕 }\end{array}$ & 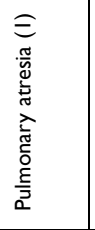 & 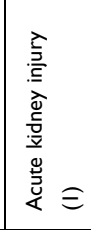 & & $\begin{array}{l}\widehat{\Xi} \\
\text { ò }\end{array}$ & & \\
\hline \multicolumn{3}{|l|}{-} & \multicolumn{3}{|l|}{-} & \multicolumn{2}{|l|}{-} & \multicolumn{2}{|l|}{-} & \multicolumn{3}{|l|}{-} & \multicolumn{3}{|l|}{-} \\
\hline 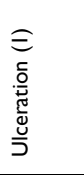 & 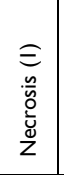 & 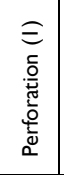 & 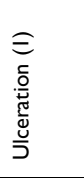 & 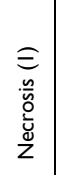 & 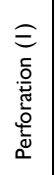 & 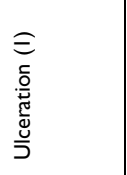 & 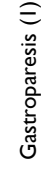 & 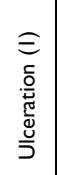 & 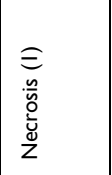 & 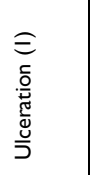 & $\begin{array}{l}\widehat{\bar{u}} \\
\frac{n}{\overline{0}} \\
\frac{\bar{u}}{\tilde{u}} \\
\bar{z}\end{array}$ & 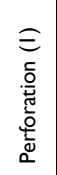 & 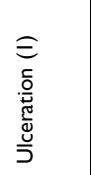 & 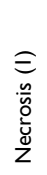 & 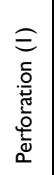 \\
\hline 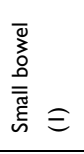 & $\begin{array}{l}\hat{0} \\
\bar{c} \\
\text { o } \\
\text { o }\end{array}$ & & 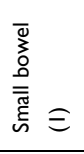 & $\begin{array}{l}\widehat{O} \\
\overline{0} \\
\text { oํㅇ } \\
0\end{array}$ & & 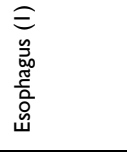 & & 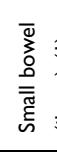 & & 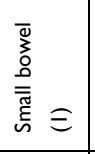 & 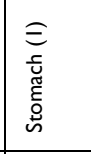 & & 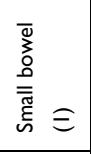 & $\begin{array}{l}\hat{\delta} \\
\frac{\delta}{0} \\
\text { o }\end{array}$ & \\
\hline 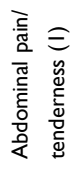 & $\begin{array}{l}\widehat{0} \\
\bar{d} \\
\frac{\mathbb{d}}{O} \\
\bar{U}\end{array}$ & & 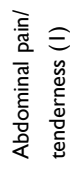 & & & 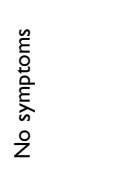 & & 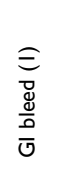 & & 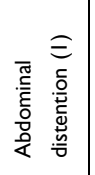 & $\begin{array}{l}\widehat{\Xi} \\
\bar{d} \\
\frac{\mathbb{J}}{0} \\
\bar{U}\end{array}$ & & 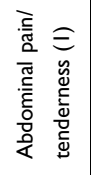 & $\begin{array}{l}\widehat{\equiv} \\
\bar{d} \\
\frac{0}{9} \\
\overline{0}\end{array}$ & \\
\hline$m$ & & & + & & & n & & - & & - & & & \pm & & \\
\hline 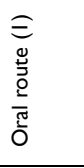 & & & 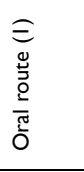 & & & 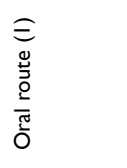 & & 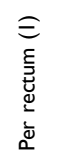 & & 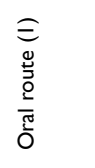 & & & 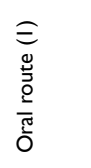 & & \\
\hline 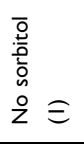 & & & $\begin{array}{l}\bar{o} \\
\frac{0}{0} \\
\overline{0} \\
\stackrel{0}{2} \\
\text { z }\end{array}$ & & & 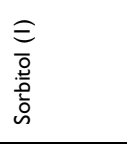 & & & & $\begin{array}{l}\overline{0} \\
\frac{9}{0} \\
\overline{0} \\
0 \\
0 \\
z\end{array}$ & & & $\begin{array}{l}\overline{0} \\
\frac{0}{0} \\
\overline{0} \\
0 \\
\frac{0}{2}\end{array}$ & & \\
\hline 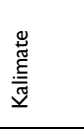 & & & 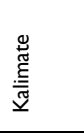 & & & 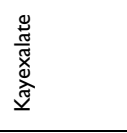 & & 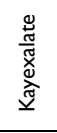 & & 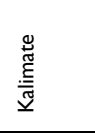 & & & 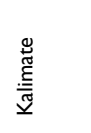 & & \\
\hline in & & & n & & & ñ & & & 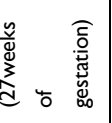 & 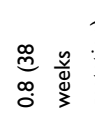 & & & $\stackrel{2}{\Omega}$ & & \\
\hline 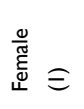 & & & 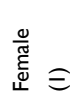 & & & 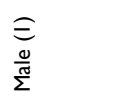 & & 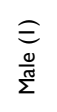 & & 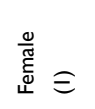 & & & 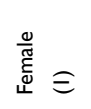 & & \\
\hline - & & & - & & & - & & - & & - & & & - & & \\
\hline 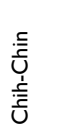 & & & 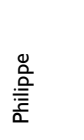 & & & 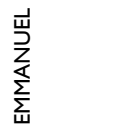 & & 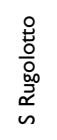 & & 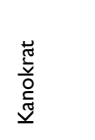 & & & $\begin{array}{l}\frac{\vec{\omega}}{\pi} \\
\frac{\pi}{3}\end{array}$ & & \\
\hline
\end{tabular}




\begin{tabular}{|c|c|c|c|c|c|c|c|c|c|c|c|c|c|c|c|c|}
\hline 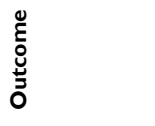 & \multicolumn{3}{|l|}{$\begin{array}{l}\widehat{\equiv} \\
\substack{0 \\
\frac{2}{<}}\end{array}$} & \multicolumn{3}{|l|}{ 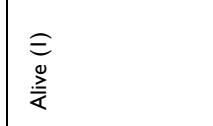 } & \multicolumn{3}{|l|}{$\begin{array}{l}\widehat{\Xi} \\
\stackrel{0}{\frac{2}{\alpha}}\end{array}$} & \multicolumn{4}{|l|}{ 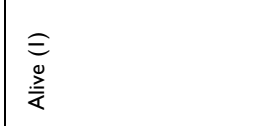 } & \multicolumn{3}{|l|}{ 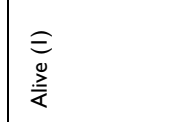 } \\
\hline 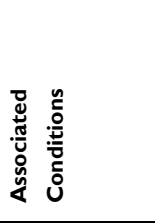 & 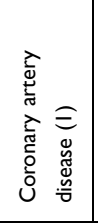 & 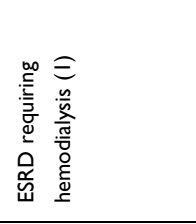 & 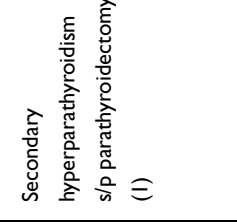 & 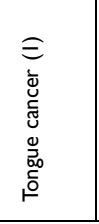 & 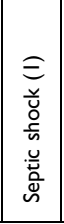 & 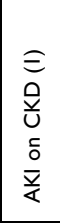 & 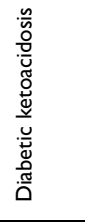 & 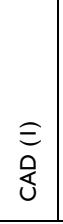 & & \begin{tabular}{|l}
$\bar{\Xi}$ \\
$\Sigma$ \\
$\Delta$
\end{tabular} & $\begin{array}{l}\text { 三 } \\
z \\
\text { z } \\
\text { I }\end{array}$ & $\begin{array}{l}\widehat{\Xi} \\
\dot{े} \\
\text { uे }\end{array}$ & $\begin{array}{l}\widehat{\Xi} \\
\text { 完 } \\
\text { I }\end{array}$ & 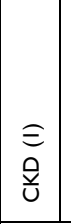 & $\begin{array}{l}\widehat{\Xi} \\
\text { 岌 } \\
\text { U }\end{array}$ & $\begin{array}{l}\text { E } \\
\text { à } \\
\text { u }\end{array}$ \\
\hline 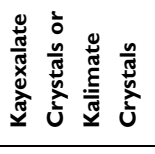 & \multicolumn{3}{|l|}{-} & \multicolumn{3}{|l|}{-} & \multicolumn{3}{|l|}{-} & \multicolumn{4}{|l|}{-} & \multicolumn{3}{|l|}{-} \\
\hline 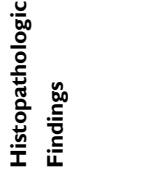 & 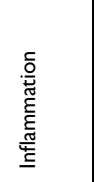 & 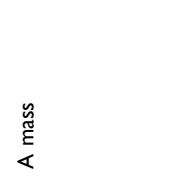 & & 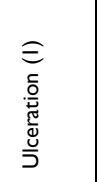 & 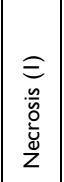 & & 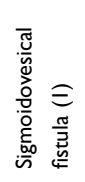 & 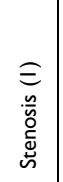 & 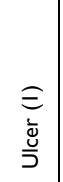 & \multicolumn{4}{|l|}{ 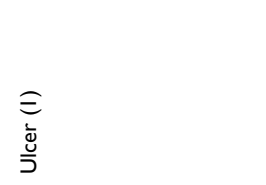 } & 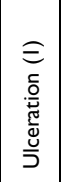 & 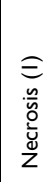 & \\
\hline لَّ & 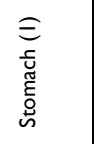 & 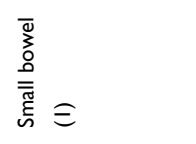 & 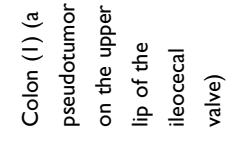 & 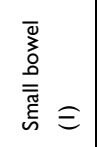 & $\begin{array}{l}0 \\
\bar{c} \\
\text { o. } \\
0\end{array}$ & & \multicolumn{3}{|l|}{$\begin{array}{l}\hat{\bar{E}} \\
\text { o. } \\
\text { O }\end{array}$} & 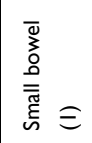 & $\begin{array}{l}1 \\
0 \\
\frac{c}{0} \\
0\end{array}$ & & & \multicolumn{3}{|l|}{$\begin{array}{l}\widehat{\equiv} \\
\text { o } \\
\text { O }\end{array}$} \\
\hline 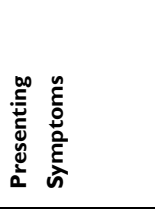 & 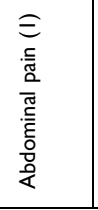 & 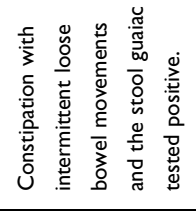 & & 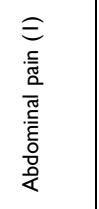 & 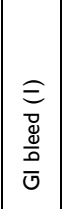 & & 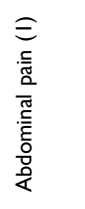 & $\begin{array}{l}\widehat{0} \\
\bar{\Xi} \\
\frac{\mathbb{\Xi}}{0} \\
\overline{0}\end{array}$ & & 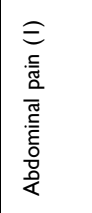 & $\begin{array}{l}\hat{\Xi} \\
\bar{\Xi} \\
\frac{\mathbb{E}}{0} \\
\bar{U}\end{array}$ & 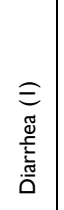 & & 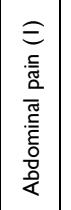 & 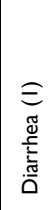 & \\
\hline 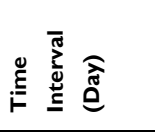 & \multicolumn{3}{|c|}{ 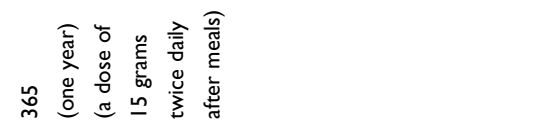 } & \multicolumn{3}{|l|}{-} & \multicolumn{3}{|l|}{$\sigma$} & \multicolumn{4}{|l|}{ 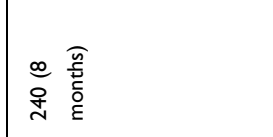 } & \multicolumn{3}{|l|}{-} \\
\hline 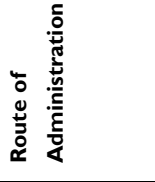 & \multicolumn{3}{|l|}{ 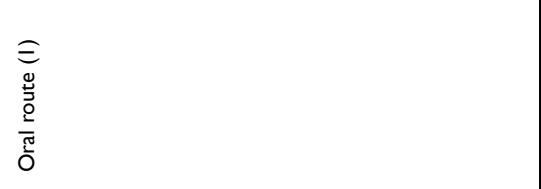 } & \multicolumn{3}{|l|}{ 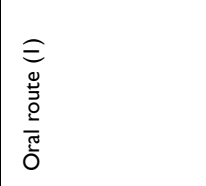 } & \multicolumn{3}{|l|}{ 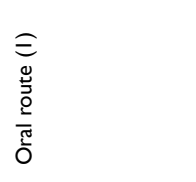 } & \multicolumn{4}{|l|}{ 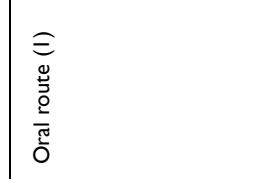 } & \multicolumn{3}{|l|}{ 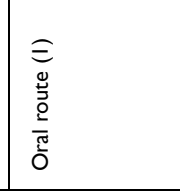 } \\
\hline 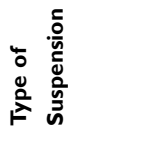 & \multicolumn{3}{|l|}{ 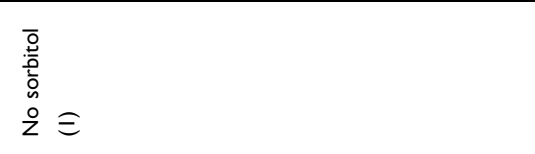 } & \multicolumn{3}{|l|}{ 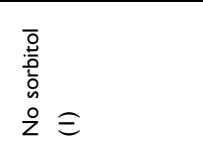 } & \multicolumn{3}{|l|}{$\begin{array}{l}\widehat{\overline{0}} \\
\overline{0} \\
\frac{0}{0} \\
\overline{0}\end{array}$} & \multicolumn{4}{|l|}{ 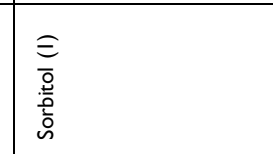 } & \multicolumn{3}{|l|}{ 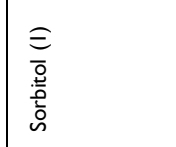 } \\
\hline 势 & \multicolumn{3}{|l|}{ 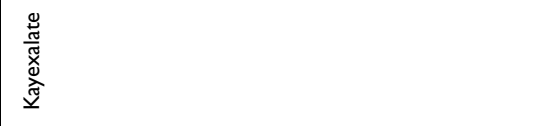 } & \multicolumn{3}{|l|}{ 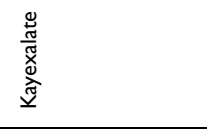 } & \multicolumn{3}{|l|}{$\begin{array}{l}\stackrel{\mathscr{\Xi}}{\tilde{E}} \\
\underline{\underline{E}} \\
\underline{\underline{E}}\end{array}$} & \multicolumn{4}{|l|}{ 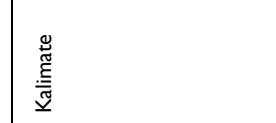 } & \multicolumn{3}{|l|}{ 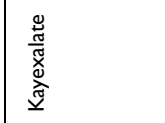 } \\
\hline 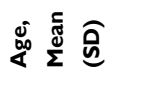 & \multicolumn{3}{|l|}{$尺$} & ț & & & 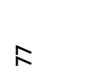 & & & $\stackrel{\circ}{R}$ & & & & \& & & \\
\hline סू & 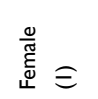 & & & $\begin{array}{l}\cong \\
\frac{0}{\tilde{c}} \\
\frac{0}{\Sigma}\end{array}$ & & & 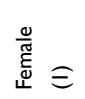 & & & 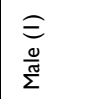 & & & & $\begin{array}{l}\frac{0}{\tilde{\pi}} \\
\underset{\varpi}{\tilde{E}}\end{array}$ & & \\
\hline 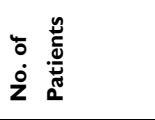 & - & & & - & & & - & & & - & & & & - & & \\
\hline 产 & 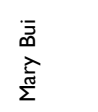 & & & 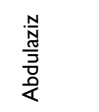 & & & $\begin{array}{l}\frac{\overline{\bar{z}}}{\overline{\underline{u}}} \\
\frac{\tilde{\omega}}{\leftarrow}\end{array}$ & & & \begin{tabular}{|l}
$\overline{\bar{\sigma}}$ \\
$\frac{\overline{\underline{g}}}{\underline{y}}$
\end{tabular} & & & & 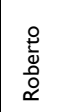 & & \\
\hline
\end{tabular}




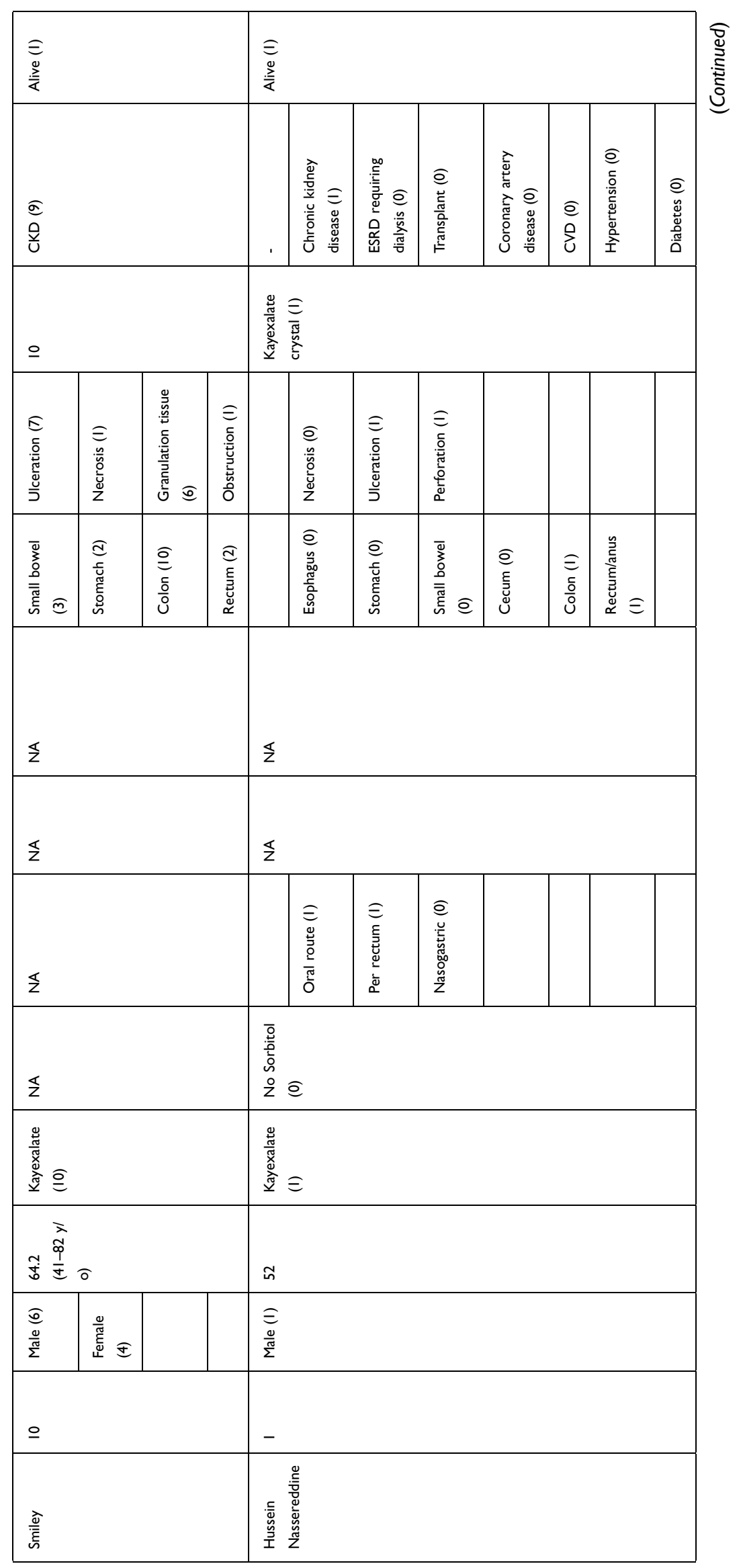




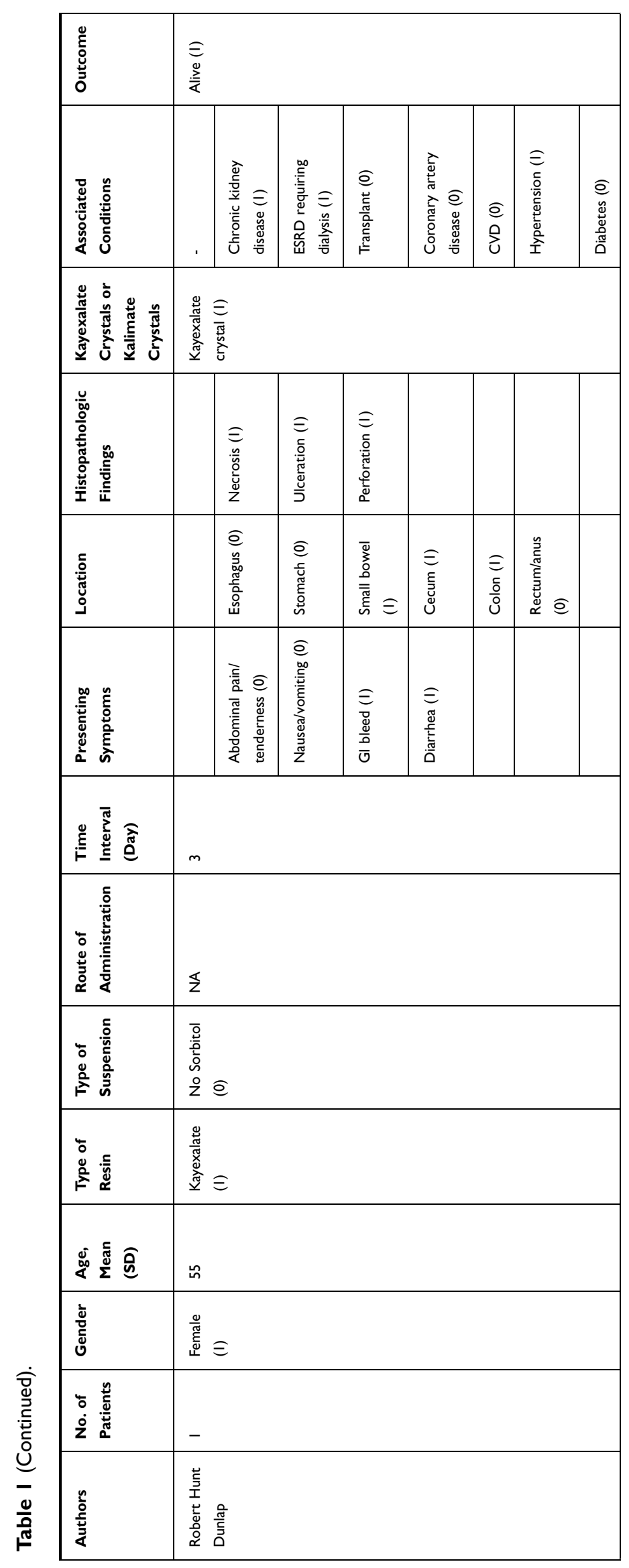




\begin{tabular}{|c|c|c|c|c|c|c|c|c|c|c|c|c|c|}
\hline $\begin{array}{l}\widehat{\mathbb{d}} \\
\stackrel{0}{\frac{2}{4}}\end{array}$ & $\begin{array}{l}\widehat{d} \\
\text { 㺃 } \\
\text { Q }\end{array}$ & & & & & & 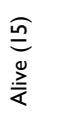 & 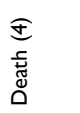 & & & & & \\
\hline 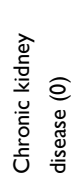 & 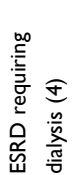 & 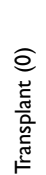 & 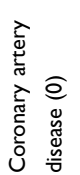 & $\begin{array}{l}0 \\
0 \\
0\end{array}$ & 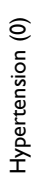 & 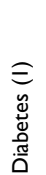 & 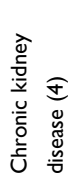 & 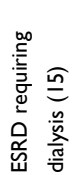 & 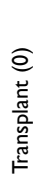 & 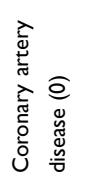 & $\begin{array}{l}0 \\
0 \\
0\end{array}$ & 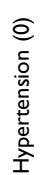 & 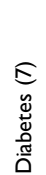 \\
\hline
\end{tabular}

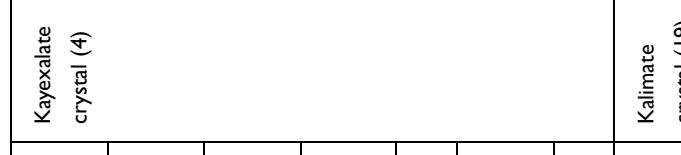

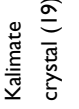

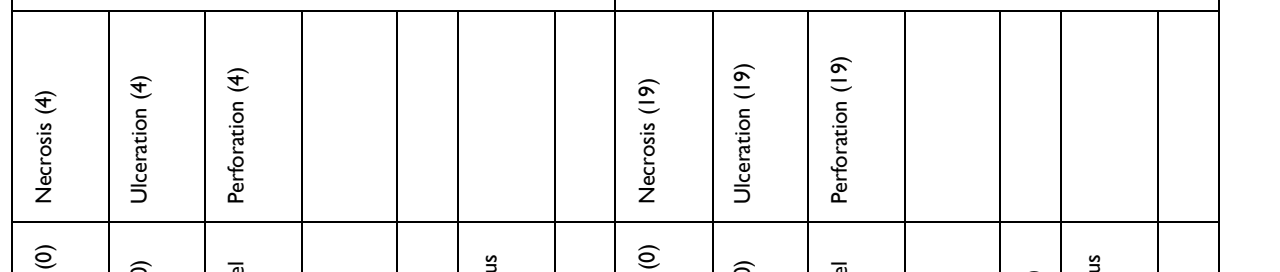

\begin{tabular}{|c|c|c|c|c|c|c|c|c|c|c|c|}
\hline 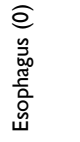 & 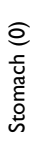 & 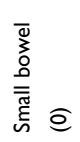 & $\begin{array}{l}\text { ô } \\
\underline{\xi} \\
\text { ư }\end{array}$ & $\begin{array}{l}\widehat{\hat{m}} \\
\bar{o} \\
\overline{0}\end{array}$ & 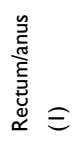 & 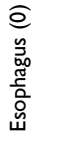 & 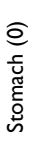 & 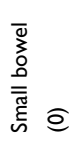 & $\begin{array}{l}\text { ô } \\
\underline{\xi} \\
\text { ư }\end{array}$ & 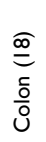 & 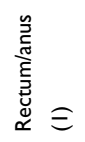 \\
\hline 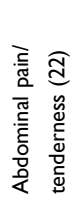 & 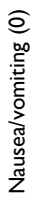 & $\begin{array}{l}\text { త్ } \\
0 \\
\frac{d}{0} \\
\frac{0}{0}\end{array}$ & 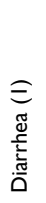 & & & 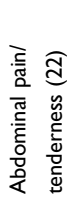 & 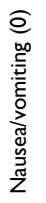 & 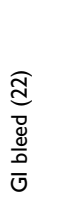 & 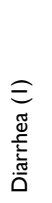 & & \\
\hline
\end{tabular}

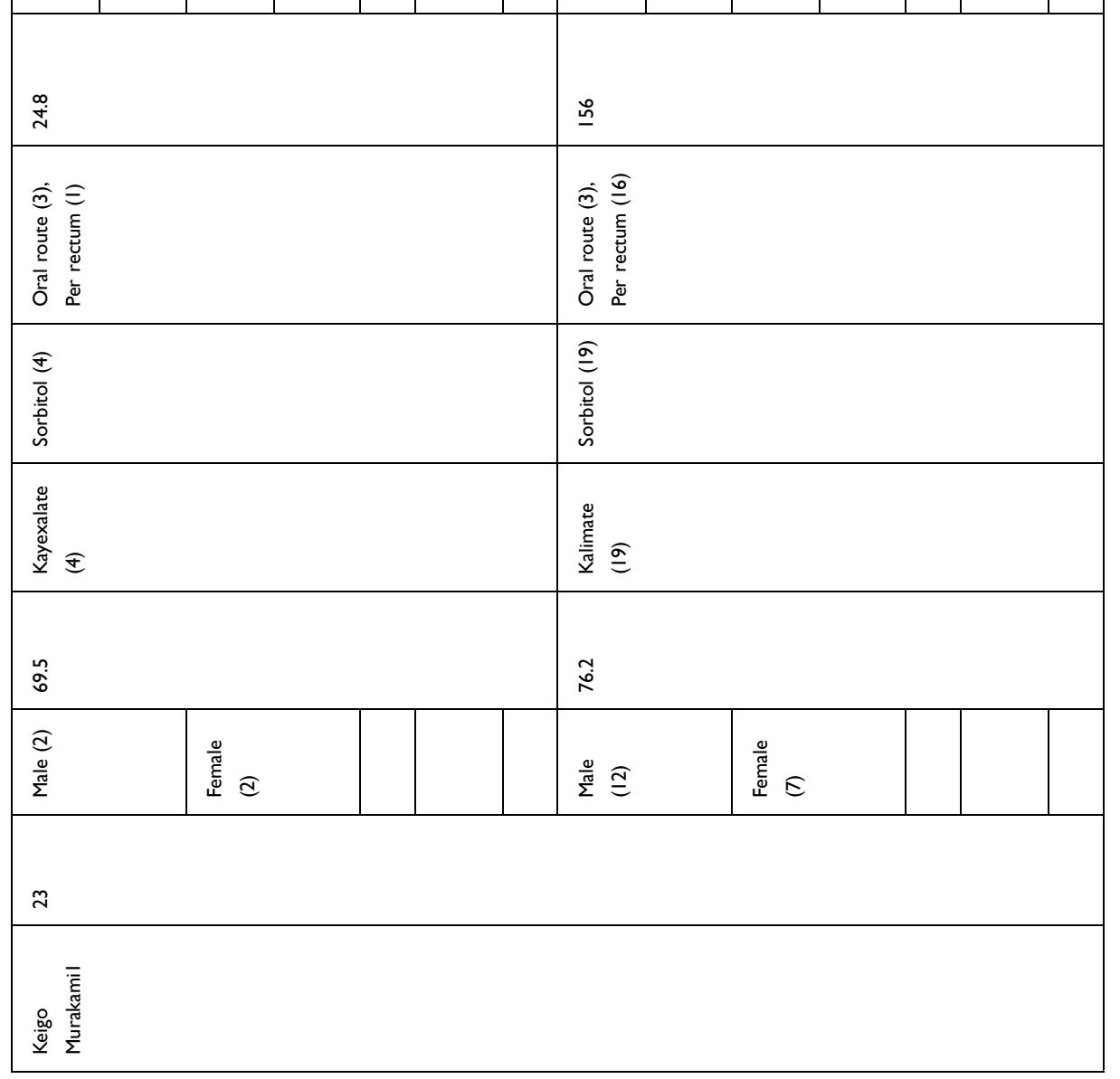




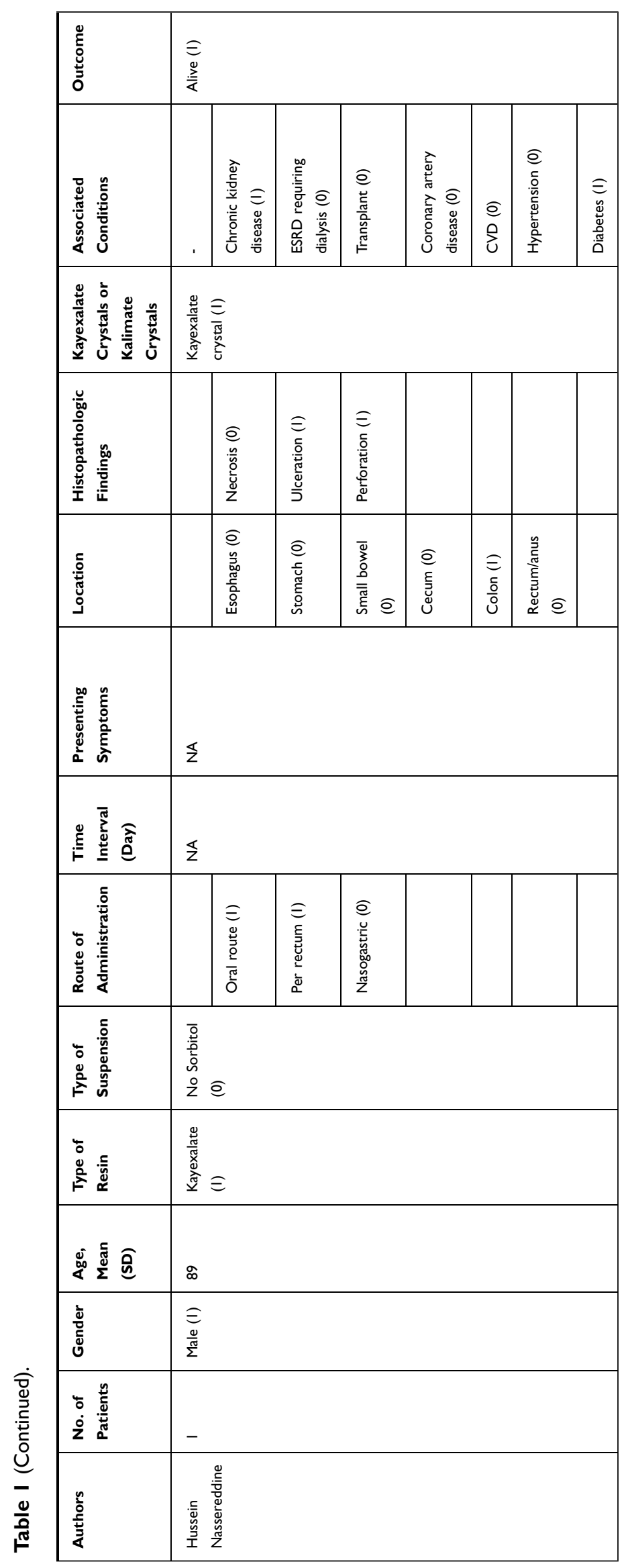


Table 2 Results of Gastrointestinal Adverse Events Associated with the Use of Kayexalate or Kalimate $(n=135)$

\begin{tabular}{|c|c|c|}
\hline Variables & Total (Valid) & $P$ value \\
\hline Presenting symptoms, n (\%) & & $<0.001$ \\
\hline Abdominal pain & $59(68.6)$ & $<0.001$ \\
\hline Nausea/vomiting & $6(7.0)$ & 0.159 \\
\hline GI bleeding & $48(55.8)$ & $<0.001$ \\
\hline Diarrhea & 18 (20.9) & 0.001 \\
\hline Location, n (\%) & & $<0.001$ \\
\hline Esophagus & $4(3.0)$ & 0.158 \\
\hline Stomach & $10(7.4)$ & 0.044 \\
\hline Small bowel & $34(25.2)$ & $<0.001$ \\
\hline Cecum & $10(7.4)$ & 0.156 \\
\hline Colon & $103(76.3)$ & $<0.001$ \\
\hline Rectum & $20(14.8)$ & $<0.001$ \\
\hline Histopathologic findings, n (\%) & & $<0.001$ \\
\hline Necrosis & $81(60.0)$ & $<0.001$ \\
\hline Ulceration & II $3(83.7)$ & $<0.001$ \\
\hline Perforation & $43(31.9)$ & 0.002 \\
\hline Kayexalate or Kalimate crystals, n (\%) & $129(95.5)$ & $<0.001$ \\
\hline Associated conditions & & $<0.001$ \\
\hline Chronic kidney disease & II 4 (87.7) & $<0.001$ \\
\hline ESRD requiring dialysis & $69(53.1)$ & $<0.001$ \\
\hline Organ transplant & $24(18.5)$ & $<0.001$ \\
\hline Coronary artery disease & $23(17.7)$ & 0.001 \\
\hline Hypertension & $39(30.0)$ & $<0.001$ \\
\hline Diabetes & $24(18.5)$ & 0.013 \\
\hline Outcome, n (\%) & & $<0.001$ \\
\hline Alive & $100(74.0)$ & \\
\hline Death & $28(20.7)$ & \\
\hline Not reported & $7(5.3)$ & \\
\hline
\end{tabular}

Abbreviations: $\mathrm{Gl}$, gastrointestinal tract; ESRD, end-stage renal disease.

the severity of the injury. The susceptibility of uremic versus non-uremic rats to injury induced by Kayexalatesorbitol enema indicates that uremia may in fact predispose to Kayexalate-induced mucosal injury. ${ }^{9}$ However, an increasing number of case reports have shown that severe adverse events, such as colonic necrosis, are linked with the Kayexalate treatment without or with only a small amount of sorbitol. ${ }^{7}$ Our present study included numerous cases using Kayexalate or Kalimate without sorbitol who experienced similar GI adverse events. ${ }^{10,11}$ Moreover, Singhania $\mathrm{N}$ et al reported a case of colonic intestinal necrosis after co-administration of Kayexalate and activated charcoal. ${ }^{12}$

Furthermore, Kayexalate or Kalimate crystals were often seen to aggregate inside the damaged parts of the GI tract specimens, examined histopathologically. The presence of Kayexalate crystals in injured GI tract segments may represent a "footprint" of its use, indicating that
Kayexalate itself may be pathogenic. ${ }^{8}$ Previous reports have postulated several risk factors for GI adverse events, including CKD, ESRD, solid organ transplantation, immunosuppression, and postoperative status; these factors lead to GI injury associated with Kayexalate or Kalimate treatment by different mechanisms. ${ }^{5,6,8,13-16,21,22}$ Renal transplant recipients are at increased risk for various adverse events, including infection, graft rejection, and medication adverse effects, in the early post-transplant period. ${ }^{8}$ Indeed, many of these risk factors were noted in most cases reviewed in the present study. Remarkably, $87.7 \%$ of all enrolled patients in this reviewing study had a history of ESRD, acute kidney injury or CKD. As shown in Table 1, the difference between these groups of comorbidities was statistically significant.

The increased renin levels, often seen in patients with renal diseases, place them at high risk to non-occlusive mesenteric ischemia via angiotensin II-mediated vasoconstriction. ${ }^{16}$ As noticed from our findings, this risk may be elevated during the postoperative period, possibly caused by ileus-induced colonic distension (resulting in lowered blood flow to colon), simultaneous hypotension and reduced gut motility as a result of opioid use, uremia, and constipation. ${ }^{5,17,18}$ Patients who recently had transplantation are at a particularly elevated risk of these complications due to the administration of immunosuppressive drugs that negatively influence the normal protective and reparative capacity of GI cells. ${ }^{18,19}$

The increase of inflammatory cytokines and prostaglandins may further impair local hemodynamic mechanisms, resulting in vascular injury and subsequent mucosal injury in the GI tract of individuals at high risk, such as those suffering with CKD or vascular disease, and patients' solid organ transplant recipients. Given our present results, the pathogenesis of GI injury related to Kayexalate or Kalimate crystals is probably more complex than what we know presently and may represent a histopathologic injury in response to different heterogeneous insults.

It is difficult to define the etiology for colonic necrosis in patients with several health issues with elevated susceptibility to mucosal injury. Combined histologic, laboratory, clinical and examinations are necessary to eliminate the commonly seen causes of mucosal necrosis and also to correctly diagnose the mucosal necrosis associated with Kayexalate- or Kalimate. ${ }^{15,18,20-22,29-53}$ However, the observation of Kayexalate or Kalimate angulated crystals with a specific mosaic pattern is critical for diagnosing 
Kayexalate- or Kalimate-related mucosal necrosis. Histologically, cholestyramine crystals (used to decrease high blood cholesterol levels or to treat itching due to biliary tract blockage) should be distinguished from Kayexalate crystals. The cholestyramine crystals are more basophilic, opaque without a mosaic pattern, and rhomboid in shape compared with the Kayexalate crystals. $^{18}$ The microscopic features of Kalimate crystals and of Kayexalate crystals are the same. ${ }^{20,22}$

The colon is the most common site of Kayexalate- or Kalimate-induced GI injuries. Injury caused by Kayexalate may appear in more proximal sections of the GI tract. ${ }^{15,18}$ As shown in Table 2, various locations of GI adverse events were statistically different, contradicting the severe adverse events in the lower GI tract from numerous case reports. Intestinal necrosis and perforation are potential complications of Kayexalate and may present in a delayed stage up to 2 months. ${ }^{28,51-53}$ However, Kayexalate- or Kalimate-related toxicities in the upper GI tract have been rarely described in the literature. ${ }^{20,22}$

Most of these cases do have simultaneous damage to colon. This change may possibly be due to the trend for the use of oral, instead of rectal Kayexalate or Kalimate. $^{5,22-28,51-53}$ However, regardless of the location of Kayexalate or Kalimate-associated injury, mortality remains high. The elevated mortality may be a result of aggravated damage along with the large comorbid disease burden of affected patients. ${ }^{5}$ In the current study, we also found safety complications associated with Kayexalate or Kalimate usage, either with or without concomitant sorbitol. ${ }^{5-53}$

This study has certain limitations. First, all results were inferred from case reports and case series. Similar to other reports concerning suspected adverse drug reactions, the relationship between Kayexalate or Kalimate and the described GI adverse events presented in our review remains uncertain. Second, this study was limited by some missing information, the absence of a standardized method of reporting adverse effects, and also possible selection and publication biases. Finally, Kayexalate- or Kalimate-associated GI adverse effects that are small in severity likely never reported and thus not recorded clinically, thereby limiting the quantification of the incidence of these events and evaluating the risk factors.

\section{Conclusions}

The use of Kayexalate or Kalimate, both with and without sorbitol, may be associated with fatal GI injuries. The most common high-risk groups of Kayexalate- or
Kalimate-induced GI injuries are patients with CKD and ESRD, followed by patients with hypertension and organ transplantations. However, Kayexalate or Kalimate is given primarily to CKD or ESRD patients because those are the patients who suffer from hyperkalemia. Therefore, it would be expected that GI adverse effects of Kayexalate or Kalimate are observed in these patients. Moreover, the most common site of GI injuries induced by Kayexalate or Kalimate is the colon. Therefore, physicians should carefully assess the patient's intestinal motility and medication history, multiple medical problems, comorbidity, and surgery before prescribing Kayexalate or Kalimate for hyperkalemia management, mainly in patients with ESRD. Physicians should also consider other safer agents when appropriate. Furthermore, detecting Kayexalate or Kalimate crystals is important for pathologists to reach a correct diagnosis.

\section{Data Sharing Statement}

Data are available from the corresponding author (Dr. JenWei Chou,codecol@yahoo.com.tw) for researchers who meet the criteria for access to confidential data.

\section{Funding}

No financial support was received for this study.

\section{Disclosure}

The authors report no conflicts of interest for this work.

\section{References}

1. Mattu A, Brady WJ, Robinson DA. Electrocardiographic manifestations of hyperkalemia. Am J Emerg Med. 2000;18(6):721-729. doi:10.1053/ajem.2000.7344

2. Iqbal Z, Friedman EA. Preferred therapy of hyperkalemia in renal insufficiency: survey of nephrology training-program directors. N Engl J Med. 1989;320:60-61.

3. Elliott MJ, Ronksley PE, Clase CM, et al. Management of patients with acute hyperkalemia. CMAJ. 2010;182(15):1631-1635. doi:10.15 03/cmaj.100461

4. Sood MM, Sood AR, Richardson R. Emergency management and commonly encountered outpatient scenarios in patients with hyperkalemia. Mayo Clin Proc. 2007;82(12):1553-1561. doi:10.101 6/S0025-6196(11)61102-6

5. Harel Z, Harel S, Shah PS, et al. Gastrointestinal adverse events with sodium polystyrene sulfonate (Kayexalate) use: a systematic review. Am J Med. 2013;126(3):264.e9. doi:10.1016/j.amjmed.2012.08.016

6. McGowan CE, Saha S, Chu G, et al. Intestinal necrosis due to sodium polystyrene sulfonate (Kayexalate) in sorbitol. South Med J. 2009;102 (5):493-497. doi:10.1097/SMJ.0b013e31819e8978

7. Gürtler N, Hirt-Minkowski P, Brunner SS, et al. Sodium polystyrene sulfonate and cytomegalovirus-associated hemorrhagic duodenitis: more than meets the eye. Am J Case Rep. 2018;19:912-916. doi:10.12659/AJCR.910655 
8. Lillemoe KD, Romolo JL, Hamilton SR, et al. Intestinal necrosis due to sodium polystyrene (Kayexalate) in sorbitol enemas: clinical and experimental support for the hypothesis.. Surgery. 1987;101(3): 267-272.

9. Ayoub I, Oh MS, Gupta R, et al. Colon Necrosis Due to Sodium Polystyrene Sulfonate with and without Sorbitol: an Experimental Study in Rats. PLoS One. 2015;10(9):e0137636. doi:10.1371/journal. pone. 0137636

10. Sterns RH, Rojas M, Bernstein P, et al. Ion-exchange resins for the treatment of hyperkalemia: are they safe and effective? J Am Soc Nephrol. 2010;21(5):733-735. doi:10.1681/ASN.2010010079

11. Watson M, Abbott KC, Yuan CM. Damned If You Do, Damned If You Don't: potassium Binding Resins in Hyperkalemia. Clin J Am Soc Nephrol. 2010;5(10):1723-1726. doi:10.2215/CJN.03700410

12. Singhania N, Al-Odat R, Singh AK, et al. Intestinal necrosis after co-administration of sodium polystyrene sulfonate and activated charcoal. Clin Case Rep. 2020;8(4):722-724. doi:10.1002/ccr3.2695

13. Gardiner GW. Kayexalate (sodium polystyrene sulphonate) in sorbitol associated with intestinal necrosis in uremic patients. Can J Gastroenterol. 1997;11(7):573-577. doi:10.1155/1997/370814

14. Montagnac R, Mehaut S, Blaison D, et al. [Colonic necrosis caused by sodium polystyrene (kayexalate) in hemodialysis: myth or reality? Two case reports].. Nephrologie. 2002;23(3):131-134.

15. Abraham SC, Bhagavan BS, Lee LA, et al. Upper gastrointestinal tract injury in patients receiving kayexalate (sodium polystyrene sulfonate) in sorbitol: clinical, endoscopic, and histopathologic findings. Am J Surg Pathol. 2001;25(5):637-644. doi:10.1097/ 00000478-200105000-00011

16. Douglas JG. Mechanism of adrenal angiotensin II receptor changes after nephrectomy in rats.. J Clin Invest. 1981;67(4):1171-1176. doi:10.1172/JCI110131

17. Dardik A, Moesinger RC, Efron G, et al. Acute abdomen with colonic necrosis induced by Kayexalate-sorbitol. South Med J. 2000;93(5):511-513. doi:10.1097/00007611-200093050-00016

18. Rashid A, Hamilton SR. Necrosis of the gastrointestinal tract in uremic patients as a result of sodium polystyrene sulfonate (Kayexalate) in sorbitol: an under recognized condition. Am J Surg Pathol. 1997;21(1):60-69. doi:10.1097/00000478-199701000-00007

19. Pirenne J, Lledo-Garcia E, Benedetti E, et al. Colon perforation after renal transplantation: a single-institution review.. Clin Transplant. 1997;11(2):88-93.

20. Joo M, Bae WK, Kim NH, et al. Colonic mucosal necrosis following administration of calcium polystryrene sulfonate (Kalimate) in a uremic patient. $J$ Korean Med Sci. 2009;24(6):1207-1211. doi:10.3346/jkms.2009.24.6.1207

21. Arvanitakis C, Malek G, Uehling D, et al. Colonic complications after renal transplantation. Gastroenterology. 1973;64(4):533-538. doi:10.1016/S0016-5085(73)80122-2

22. Wu YH, Chen TW, Chou JW. Kalimate-induced upper gastrointestinal bleeding in a 77-year-old woman. Clin Gastroenterol Hepatol. 2019;S1542(19):30516.

23. Su G-S, Chen T-W, Chou J-W. A rare cause of abdominal pain with bloody stool. Turk J Gastroenterol. 2020;31(1):73-74. doi:10.5152/ tjg.2020.18821

24. Piron L, Ramos J, Taourel P. Colonic perforation associated with sodium polystyrene sulfonate (Kayexalate) use. Diagn Interv Imaging. 2018;99(7):507-509. doi:10.1016/j.diii.2018.02.003

25. Evans BM, Milne MD, Hughes Jones NC, et al. Ion-exchange resins in the treatment of anuria. Lancet. 1953;262(6790):791-795. doi:10.1016/S0140-6736(53)90465-6

26. Flinn RB, Merrill JP, Welzant WR. Treatment of the oliguric patient with a new sodium-exchange resin and sorbitol; a preliminary report. $N$ Engl J Med. 1961;264(3):111-115. doi:10.1056/NEJM196101192640302

27. Scherr L, Ogden DA, Mead AW, et al. Management of hyperkalemia with a cation-exchange resin. $N$ Engl J Med. 1961;264(3):115-119. doi:10.1056/NEJM196101192640303
28. Bogaerts JMA, van der Hoeven JG, Arts EEA, et al. Fish scale crystals: an under-recognised cause of intestinal necrosis. J Clin Pathol. 2019;72(8):567. doi:10.1136/jclinpath-2018-205203

29. Yung WT, Chan SY. Recurrent intestinal necrosis due to polystyrene sulphonate use - a case report. AJCRS. 2019;2:1-4.

30. Tongyoo A, Sriussadaporn E, Limpavitayaporn P, et al. Acute intestinal obstruction due to Kalimate, a potassium-lowering agent: a case report and literature review.. J Med Assoc Thai. 2013;96(12):1617-1620.

31. Patel S, Arnold CA, Gray DM. A case of sodium polystyrene sulfonate (Kayexalate)-induced bowel ischemia: A reminder of adverse effects of a common medication. Am J Gastroenterol. 2017;112: S1365. doi:10.14309/00000434-201710001-02502

32. Ribeiro H, Pereira E, Banhudo A. Colonic necrosis induced by calcium polystyrene sulfonate. GE - Portuguese Journal of Gastroenterology. 2018;25(4):205-207. doi:10.1159/000481288

33. Ponroy B, Nadal M, Nardoux J, et al. Ulcérations coliques associées à la prise de Kayexalate ${ }^{\circledR}$ (polystyrène sulfonate de sodium): à propos de deux observations. Ann Pathol. 2015;35(2):164-167. doi:10.1016/ j.annpat.2015.01.013

34. Panarelli NC. Drug-induced injury in the gastrointestinal tract. Semin Diagn Pathol. 2014;31(2):165-175. doi:10.1053/j.semdp.2014.02.007

35. Nuzzo A, Shaar-Chneker C, Maillet M, et al. Gastroduodenal injury induced by orally administered sodium polystyrene sulfonate. Clin Toxicol. 2019;57(1):75-76. doi:10.1080/15563650.2018.1518529

36. Say DS, Starnes T, Matsukuma K, et al. Gastrointestinal bleeding after hemolytic uremic syndrome: a report of drug-associated granulation tissue polyps. J Pediatr Gastroenterol Nutr. 2018;67(5):e102. doi:10.1097/MPG.0000000000002044

37. Kathleen S, Elena M, Meaghen F, et al. Hyperkalemia management in the oncology patient: a case of sodium polystyrene sulfate induced bowel perforation. J Anest Inten Care Med. 2017;3:555610.

38. McCarthy AJ, Lauwers GY, Sheahan K. Iatrogenic pathology of the intestines. Histopathology. 2015;66(1):15-28. doi:10.1111/his.12598

39. Kao -C-C, Tsai Y-C, Chiang W-C, et al. Ileum and colon perforation following peritoneal dialysis-related peritonitis and high-dose calcium polystyrene sulfonate. J Formos Med Assoc. 2015;114 (10):1008-1010. doi:10.1016/j.jfma.2013.02.006

40. Goutorbe P, Montcriol A, Lacroix G, et al. Intestinal necrosis associated with orally administered calcium polystyrene sulfonate without sorbitol. Ann Pharmacother. 2011;45(2):e13. doi:10.1345/aph.1M547

41. Gorospe EC, Lewis JT, Bruining DH. Kayexalate-induced esophageal ulcer in a patient with gastroparesis. Clin Gastroenterol Hepatol. 2012;10(5):A28. doi:10.1016/j.cgh.2011.12.026

42. Chen Z, Scudiere JR, Montgomery E. Medication-induced upper gastrointestinal tract injury. J Clin Pathol. 2009;62(2):113-119. doi:10.1136/jcp.2008.058263

43. Rugolotto S, Gruber M, Solano PD, et al. Necrotizing enterocolitis in a 850 gram infant receiving sorbitol-free sodium polystyrene sulfonate (Kayexalate): clinical and histopathologic findings. J Perinatol. 2007;27(4):247-249. doi:10.1038/sj.jp.7211677

44. Thaiwatcharamas K, Phannua R, Chusilp S, et al. Neonatal gastric necrosis and perforation associated with impacted medication. J Pediatr Surg Case Rep. 2019;42:45-47. doi:10.1016/j.epsc.2018. 12.024

45. Bui M, Chou S-Y, Faubert P, et al. Resin-Induced Colonic Pseudotumor: rare Complication from Chronic Use of Potassium Binders in a Hemodialysis Patient.. Case Rep Nephrol. 2016;2016:3692086. doi:10.1155/2016/3692086

46. Almulhim AS, Hall E, Rehaili B, et al. Sodium polystyrene sulfonate induced intestinal necrosis; a case report. Saudi Pharm J. 2018;26 (6):771-774. doi:10.1016/j.jsps.2018.04.008

47. Shioya T, Yoshino M, Ogata M, et al. Successful treatment of a colonic ulcer penetrating the urinary bladder caused by the administration of calcium polystyrene sulfonate and sorbitol. Journal of Nippon Medical School. 2007;74(5):359-363. doi:10.1272/jnms. 74.359 
48. Okayama K, Hirata Y, Kumai D, et al. The Successful Treatment of Sodium Polystyrene Sulfonate-induced Enteritis Diagnosed by Small Bowel Endoscopy. Intern Med. 2018;57(11):1577-1581. doi:10.2169/ internalmedicine.0088-17

49. Cerrud-Rodriguez RC, Alcaraz-Alvarez D, Chiong BB, et al. Vancomycin-resistant Enterococcus faecium bacteraemia as a complication of Kayexalate (sodium polystyrene sulfonate, SPS) in sorbitol-induced ischaemic colitis. BMJ Case Rep. 2017;2017: bcr2017221790. doi:10.1136/bcr-2017-221790

50. Georgianos PI, Liampas I, Kyriakou A, et al. Evaluation of the tolerability and efficacy of sodium polystyrene sulfonate for long-term management of hyperkalemia in patients with chronic kidney disease. Int Urol Nephrol. 2017;49(12):2217-2221. doi:10.1007/s11255-017-1717-5
51. Dunlap RH, Martinez R. Total colectomy for colon perforation after kayexalate administration: a case report and literature review of a rare complication. J Surg Case Rep. 2016;10:1-3.

52. Murakami K, Nakamura Y, Miyasaka Y, et al. Intestinal necrosis related to administration of cation exchange resin without sorbitol: A retrospective analysis of 61 patients with end-stage renal diseases. Pathol Int. 2020;70(5):270-9. doi:10.1111/pin.12906

53. Nassereddine H, Pelletier A-L, Chemouny JM, et al. Risk of perforated colonic diverticulitis in patients with chronic kidney disease requiring sodium polystyrene sulfonate: not to Be Forgotten. $\mathrm{Am}$ $J$ Gastroenterol. 2019;114(6):1003-5. doi:10.14309/ajg.000000000 0000276

\section{Publish your work in this journal}

Clinical and Experimental Gastroenterology is an international, peerreviewed, open access, online journal publishing original research, reports, editorials, reviews and commentaries on all aspects of gastroenterology in the clinic and laboratory. This journal is indexed on American Chemical Society's Chemical Abstracts Service (CAS).
The manuscript management system is completely online and includes a very quick and fair peer-review system, which is all easy to use. Visit http://www.dovepress.com/testimonials.php to read real quotes from published authors. 